\title{
Contragenic Functions on Spheroidal Domains
}

\author{
R. García Ancona ${ }^{a}$, J. Morais ${ }^{b}$ and R. Michael Porter ${ }^{a}$ \\ ${ }^{a}$ Department of Mathematics, CINVESTAV-Querétaro, Apdo. Postal 1-798, Arteaga 5, \\ 76000 Santiago de Querétaro, Qro., Mexico. \\ ${ }^{b}$ Department of Mathematics, ITAM, Rio Hondo \#1, Col. Progreso Tizapán, México, \\ DF 01080, México.E-mail: joao.morais@itam.mx
}

\begin{abstract}
We construct bases of polynomials for the spaces of square-integrable harmonic functions which are orthogonal to the monogenic and antimonogenic $\mathbb{R}^{3}$-valued functions defined in a prolate or oblate spheroid.

Keywords: quaternionic analysis; monogenic function; hyperholomorphic function; contragenic function; spheroidal harmonics.
\end{abstract}

\section{Introduction}

The theory of holomorphic functions in Clifford algebras, and in particular quaternions, is quite extensive [2, 12, 30]. In recent years the corresponding results for quaternionic-differentiable functions defined in domains of $\mathbb{R}^{3}$ have been developed [3, 5, 6, 7, 8, 10, 14, 22, 23, 25, 27, 31] with a view to making this theory applicable to physical systems. In particular, a function from $\mathbb{R}^{3}$ to $\mathbb{R}^{3}$ is quaternion-holomorphic (monogenic) precisely when it satisfies the Riesz system of partial differential equations [9, 15, 17, 18, 19].

Spheres are commonly used as the reference domain for modeling physical problems. However, in many cases, a spheroidal domain may offer a better approximation to reality. Here we study the natural basis of harmonic polynomials in $\operatorname{Har}_{2}(\Omega)=L_{2}(\Omega) \cap \operatorname{Har}(\Omega)$ where $\Omega$ is a prolate or oblate spheroid. Our attention is directed to what are now known as contragenic functions, which are orthogonal to all monogenic and antimonogenic $L_{2}$ harmonic functions. Previous to [1] the existence of such functions was not suspected. It is necessary to understand the contragenic functions in order to be able to consider the "monogenic part" of a given harmonic function. Contragenicity, 
in contrast to harmonicity and monogenicity, is not a local property, since it depends on the domain under consideration.

In Section 2 the spheroidal harmonics are defined following [11], with a rescaling factor which permits including the sphere as a limit of both the prolate and oblate cases, combined into a single one-parameter family. The spheroidal monogenic polynomials are calculated in Section 3, and new explicit formulas for their nonscalar parts are obtained in terms of the spheroidal harmonics. A basis for the space of functions obtained by summing a monogenic function with an antimonogenic function is written out. All of these orthogonal bases are composed of elements parametrized by the shape of the corresponding spheroid, and their norms are calculated explicitly. In the final section we produce an orthogonal basis for the contragenic spheroidal polynomials.

\section{Spheroidal harmonics}

Analysis of harmonic and monogenic functions on spheroids (cf. 11, 13, 16, 20, 21, 24, 26, 29] typically separates the prolate and oblate cases, parametrized in their respective confocal families

$$
\begin{aligned}
& \left\{x \in \mathbb{R}^{3} \mid \frac{x_{0}^{2}}{\cosh ^{2} \alpha}+\frac{x_{1}^{2}+x_{2}^{2}}{\sinh ^{2} \alpha}<1\right\}, \\
& \left\{x \in \mathbb{R}^{3} \mid \frac{x_{0}^{2}}{\sinh ^{2} \alpha}+\frac{x_{1}^{2}+x_{2}^{2}}{\cosh ^{2} \alpha}<1\right\},
\end{aligned}
$$

for $\alpha>0$. These domains do not include the case of a Euclidean ball (where harmonic analysis originated), but they become rounder as they degenerate with $\alpha \rightarrow \infty$. Thus we prefer to combine them into a single family

$$
\Omega_{\mu}=\left\{x \in \mathbb{R}^{3} \mid x_{0}^{2}+\frac{x_{1}^{2}+x_{2}^{2}}{e^{2 \nu}}<1\right\},
$$

where $\nu \in \mathbb{R}$ is arbitrary, and $\mu=\left(1-e^{2 \nu}\right)^{\frac{1}{2}}$ by convention is in the interval $(0,1)$ when $\nu<0$ (prolate spheroid), and in $i \mathbb{R}^{+}$when $\nu>0$ (oblate spheroid); the intermediate value $\nu=0, \mu=0$ gives the unit ball $\Omega_{0}=\left\{x:|x|^{2}<1\right\}$. The convenience of the parameter $\mu$ will become evident later. Here we note that in the prolate case, we obtain $\Omega_{\mu}$ by setting $e^{\nu}=\tanh \alpha$ and rescaling $x$ by a factor of $\mu^{-1}$, while for the oblate case, we set $e^{\nu}=\operatorname{coth} \alpha$ and rescale by a factor of $(\mu / i)^{-1}$. 
The spheroidal harmonics $U_{n, m}^{ \pm}[\mu]$ of [11] are constructed as follows. Suppose first that $\nu<0$. For this case we use coordinates $(u, v, \phi)$ defined on the prolate spheroid by

$$
x_{0}=\mu \cos u \cosh v, \quad x_{1}=\mu \sin u \sinh v \cos \phi, \quad x_{2}=\mu \sin u \sinh v \sin \phi \text {, }
$$

where $u \in[0, \pi], v \in\left[0, \operatorname{arctanh} e^{\nu}\right], \phi \in[0,2 \pi]$, and $\mu>0$. Then for $x \in \Omega_{\mu}$ we define

$$
U_{n, m}^{ \pm}[\mu](x)=\alpha_{n, m} \mu^{n} P_{n}^{m}(\cos u) P_{n}^{m}(\cosh v) \Phi_{m}^{ \pm}(\phi),
$$

where

$$
\alpha_{n, m}=\frac{(n-m) !}{(2 n-1) ! !}
$$

(with use of the symbol $n ! !=\prod_{k=0}^{\lceil n / 2\rceil-1}(n-2 k)$ for the double factorial) and we have written

$$
\Phi_{m}^{+}(\phi)=\cos (m \phi), \quad \Phi_{m}^{-}(\phi)=\sin (m \phi)
$$

in order to unify the notation for the odd and even functions. Here $P_{n}^{m}$ denotes the associated Legendre function of the first kind [13, Ch. III], of degree $n$ and order $m$.

Observe that $\cos u=2 x_{0} / \omega$ and $\cosh v=\omega /(2 \mu)$, where

$$
\omega=\sqrt{\left(x_{0}+\mu\right)^{2}+x_{1}^{2}+x_{2}^{2}}+\sqrt{\left(x_{0}-\mu\right)^{2}+x_{1}^{2}+x_{2}^{2}}
$$

is positive. The oblate case $\nu>0$ is obtained from this by analytic continuation, thinking of $\mu \in i \mathbb{R}^{+}$as being boundary values of the first quadrant in complex plane. The terms $\zeta=\left(|x|^{2}+\mu^{2}\right)+2 x_{0} \mu$ and $\bar{\zeta}$ inside the radicals in (66) are now complex conjugates, so $\omega=\sqrt{2(|\zeta|+\operatorname{Re} \zeta)}$ is real and slightly less than $2|x|$ for $\mu / i$ small. Then

$$
\frac{2 x_{0}}{\omega}=x_{0} \sqrt{\frac{2}{|\zeta|+\operatorname{Re} \zeta}}, \quad \frac{\omega}{2 \mu}=i \sqrt{\frac{|\zeta|+\operatorname{Re} \zeta}{2\left(e^{2 \nu}-1\right)}},
$$

and one can verify from this that $\left|2 x_{0} / \omega\right| \leq 1$ and that $\operatorname{Im}(\omega / 2 \mu)$ takes values in $[0, \infty)$.

Consequently, for an oblate spheroid we replace the coordinate $v$ with the value $\operatorname{arcsinh} \cosh v$ in order to retain formula (2) via the relations $2 x_{0} / \omega=$ 
$\cos u$ and $\omega /(2 \mu)=i \sinh v$, with $u \in[0, \pi]$ and $v \in\left[0\right.$, arccoth $\left.e^{\nu}\right]$, and use (3) again to define the oblate harmonics. This completes the construction of the spheroidal harmonics.

Note that $U_{n, 0}^{-}[\mu]$ vanishes identically, as do all $U_{n, m}^{ \pm}[\mu]$ for $m \geq n+1$. Therefore when we refer to the set $\left\{U_{n, m}^{ \pm}[\mu]\right\}$ we always exclude the indices which refer to these trivial cases, even when we do not state explicitly $0 \leq$ $m \leq n$ for the "+" case and $1 \leq m \leq n$ for the "-" case. In general the polynomials $U_{n, m}^{ \pm}[\mu]$ are not homogeneous, unlike the classical spherical harmonics [28]

$$
U_{n, m}^{ \pm}[0]=|x|^{n} P_{n}^{m}\left(\frac{x_{0}}{|x|}\right) \Phi_{m}^{ \pm}(\phi)
$$

where $x_{0}=r \cos \theta, x_{1}=r \sin \theta \cos \phi, x_{2}=r \sin \theta \sin \phi$.

The family $\left\{U_{n, m}^{ \pm}[\mu]\right\}$ turns out to be orthogonal with respect to the Dirichlet inner product [11], but not in $L_{2}\left(\Omega_{\mu}\right)$. Define

$$
V_{n, m}^{ \pm}[\mu](x)=\frac{\partial}{\partial x_{0}} U_{n+1, m}^{ \pm}[\mu](x) .
$$

Since the functions (8) , except for the constant factors $\alpha_{n, m}$ and the rescaling of the $x$ variable, are the functions defined in [11], the main result of that paper can be restated as follows.

Theorem 2.1. The functions $V_{n, m}^{ \pm}[\mu](n \geq 0)$ are harmonic polynomials in $x_{0}, x_{1}, x_{2}$ of degree $n$. They form a complete orthogonal family in the closed subspace $L_{2}\left(\Omega_{\mu}\right) \cap \operatorname{Har}\left(\Omega_{\mu}\right)$ of $L_{2}\left(\Omega_{\mu}\right)$. Furthermore,

$$
\left\|V_{n, m}^{ \pm}[\mu]\right\|_{2}^{2}=\left(1+\delta_{0, m}\right) \mu^{2 n+3} \beta_{n, m} \int_{1}^{\frac{1}{\mu}} P_{n}^{m}(t) P_{n+2}^{m}(t) d t,
$$

where $\delta_{m, m^{\prime}}$ is the Kronecker symbol, and

$$
\beta_{n, m}=\frac{\pi 2^{n+1}(n+m+1)(n-m+2) !(n+m+1) !}{(2 n+1) ! !(2 n+3) ! !} .
$$

The use of the particular coefficient $\alpha_{n, m}$ in (3) is for the following.

Proposition 2.2. For every $x \in \mathbb{R}^{3}$, the limit $\lim _{\mu \rightarrow 0} V_{n, m}^{ \pm}[\mu](x)$ exists and is given by $V_{n, m}^{ \pm}[0](x)=\left(\partial / \partial x_{0}\right) U_{n+1, m}^{ \pm}[0](x)$, where $U_{n+1, m}^{ \pm}[0]$ is the classical spherical harmonic (cf. (77)). 
Proof. It is sufficient to prove that $U_{n, m}^{ \pm}[\mu] \rightarrow U_{n, m}^{ \pm}[0]$. Since $\phi$ in (2) and (7) does not depend on $x_{0}$, we examine the factors $P_{n}^{m}\left(2 x_{0} / \omega\right) P_{n}^{m}(\omega /(2 \mu))$ in (3), with $\omega$ again given by (6). Since

$$
\sqrt{\left(x_{0} \pm \mu\right)^{2}+x_{1}^{2}+x_{2}^{2}}=|x| \pm\left(x_{0} /|x|\right) \mu+O\left(\mu^{2}\right),
$$

we have $\omega=2|x|+O\left(\mu^{2}\right)$ as $\mu \rightarrow 0$.

A direct computation using (6) shows that $2 x_{0} / \omega=x_{0} /|x|+O(\mu)$, so $P_{n}^{m}\left(2 x_{0} / \omega\right) \rightarrow P_{n}^{m}\left(x_{0} / x\right)$ as $\mu \rightarrow 0$. It can be shown inductively that $\alpha_{n, m}=$ $2^{-n} n !(n+m) ! \sum_{k=m}^{n} \lambda_{k}^{n, m}$, where $\lambda_{k}^{n, m}=((n+m-k) !(n-k) !(k-m) ! k !)^{-1}$. From the explicit representation

$$
P_{n}^{m}(t)=\frac{n !(m+n) !}{2^{n}}\left(t^{2}-1\right)^{m / 2} \sum_{k=m}^{n} \lambda_{k}^{n, m}(t-1)^{n-k}(t+1)^{k-m}
$$

valid for real $|t|>1$, we have the required asymptotic behavior

$$
P_{n}^{m}(t) \simeq \frac{1}{\alpha_{n, m}} t^{n}
$$

as $t=\omega / 2 \mu$ tends to infinity, which corresponds to $\mu \rightarrow 0$ for fixed $x$.

The spherical harmonics are embedded in this 1-parameter family of spheroidal harmonics. In contrast, in treatments such as [11, 13, 16, 20, 24, 26, 29], the spheroidal harmonics degenerate to a segment as the eccentricity of the spheroid decreases.

We introduce the notation $V_{n, m}^{ \pm}[\mu]=\widehat{V}_{n, m}[\mu] \Phi_{m}^{ \pm}, U_{n, m}^{ \pm}[\mu]=\widehat{U}_{n, m}[\mu] \Phi_{m}^{ \pm}$for use when the factors $\Phi_{m}^{ \pm}$are not of interest. The following will be key in the proof of Theorem 3.2 and it is based on the results of [16].

Proposition 2.3. For each $n \geq 2$, the functions $\widehat{V}_{n, m}[\mu]$ satisfy the recurrence relation

$$
\widehat{V}_{n, m}[\mu]=(n+m+1) \widehat{U}_{n, m}[\mu]+\frac{\mu^{2}(n+m+1)(n+m)}{(2 n+1)(2 n-1)} \widehat{V}_{n-2, m}[\mu] .
$$

Proof. We will assume that $\nu<0$, because the case $\nu>0$ is similar. From differentiating (2),

$$
\frac{\partial}{\partial x_{0}}=\frac{1}{\mu\left(\cos ^{2} u-\cosh ^{2} v\right)}\left(\sin u \cosh v \frac{\partial}{\partial u}-\cos u \sinh v \frac{\partial}{\partial v}\right),
$$


from which the definition (8) gives

$$
\begin{aligned}
\frac{\left(\cos ^{2} u-\cosh ^{2} v\right)}{\alpha_{n+1, m} \mu^{n}} \widehat{V}_{n, m}= & \left(-\sin ^{2} u \cosh v P_{n+1}^{m}(\cosh v)\left(P_{n+1}^{m}\right)^{\prime}(\cos u)\right. \\
& \left.-\cos u \sinh ^{2} v P_{n+1}^{m}(\cos u)\left(P_{n+1}^{m}\right)^{\prime}(\cosh v)\right)
\end{aligned}
$$

There are many well-known recurrence relations for the associated Legendre functions (see for example [13, Ch. III]). The relation

$$
\left(1-t^{2}\right)\left(P_{n+1}^{m}\right)^{\prime}(t)=(n+m+1) P_{n}^{m}(t)-(n+1) t P_{n+1}^{m}(t)
$$

yields that (11) is equal to $(n+m+1)$ times

$$
\cosh v P_{n}^{m}(\cos u) P_{n+1}^{m}(\cosh v)-\cos u P_{n+1}^{m}(\cos u) P_{n}^{m}(\cosh v) .
$$

The further relation

$$
(n-m+1) P_{n+1}^{m}(t)=(2 n+1) t P_{n}^{m}(t)-(n+m) P_{n-1}^{m}(t)
$$

shows that

$$
\begin{aligned}
\widehat{V}_{n, m}= & (n+m+1) \widehat{U}_{n, m} \\
& +\frac{\alpha_{n, m} \mu^{n}(n+m+1)(n+m)}{\left(\cosh ^{2} v-\cos ^{2} u\right)(2 n+1)}\left[\cos u P_{n-1}^{m}(\cos u) P_{n}^{m}(\cosh v)\right. \\
& \left.-\cosh v P_{n}^{m}(\cos u) P_{n-1}^{m}(\cosh v)\right]
\end{aligned}
$$

as is seen after substituting

$$
\alpha_{n, m}=\left(\frac{2 n+1}{n-m+1}\right) \alpha_{n+1, m} .
$$

Using (14) again, straightforward computations show that

$$
\begin{gathered}
\widehat{V}_{n, m}=(n+m+1) \widehat{U}_{n, m} \\
+\frac{\alpha_{n-1, m} \mu^{n}(n+m+1)(n+m)(n+m-1)}{\left(\cosh ^{2} v-\cos ^{2} u\right)(2 n-1)(2 n+1)} \times \\
{\left[\cosh v P_{n-2}^{m}(\cos u) P_{n-1}^{m}(\cosh v)\right.} \\
\left.-\cos u P_{n-1}^{m}(\cos u) P_{n-2}^{m}(\cosh v)\right] .
\end{gathered}
$$

The result now follows. 


\section{Spheroidal monogenic functions}

Regard $\mathbb{R}^{3}$ as the subset of the quaternions $\mathbb{H}=\left\{x_{0}+x_{1} e_{1}+x_{2} e_{2}+x_{3} e_{3}\right\}$ for which $x_{3}=0$. Although this subspace is not closed under quaternionic multiplication (which is defined, as is usual, so that $e_{i}^{2}=-1$ and $e_{i} e_{j}=-e_{j} e_{i}$ for $i \neq j$ ), it is possible to carry out a great deal of the analysis analogous to that of complex numbers $[9,12,15,17,18,19]$.

Consider the Cauchy-Riemann (or Fueter) operators

$$
\partial=\frac{\partial}{\partial x_{0}}-\sum_{i=1}^{2} \frac{\partial}{\partial x_{i}}, \quad \bar{\partial}=\frac{\partial}{\partial x_{0}}+\sum_{i=1}^{2} \frac{\partial}{\partial x_{i}} .
$$

A smooth function $f$ defined in an open set of $\mathbb{R}^{3}$ is (left-)monogenic when $\partial f=0$, and (left-)antimonogenic when $\bar{\partial} f=0$ identically. The term "hyperholomorphic" is also commonly used.

\subsection{Construction of orthogonal basis of monogenics}

A basis of polynomials spanning the square-integrable solutions of $\bar{\partial} f=0$ was given in [16] and another in [20] for prolate spheroids, via explicit formulas. However, following [11] here we take another approach to monogenic functions, more suitable to our purposes. We consider simultaneously the prolate and oblate cases of spheroids. Define the basic monogenic spheroidal polynomials to be

$$
X_{n, m}^{ \pm}[\mu]=\partial U_{n+1, m}^{ \pm}[\mu] .
$$

They are indeed monogenic since $U_{n+1, m}^{ \pm}[\mu]$ is harmonic, in view of the factorization $\Delta=\bar{\partial} \partial$ of the Laplacian. Now we will work out explicit expressions in terms of the orthogonal basis of harmonic functions; some examples in low degree are exhibited in Tables 1 and 2 .

As was shown in [16], the equality

$$
\widehat{V}_{n,-1}=-\frac{1}{(n+1)(n+2)} \widehat{V}_{n, 1}
$$

can be easily verified for $n \geq 0$. These functions will appear in the representation (18) for the case of zero-order monogenic polynomials (see Theorem 3.1 below). 
Theorem 3.1. For each $n \geq 0$ and $0 \leq m \leq n+1$, the basic spheroidal monogenic polynomial is equal to

$$
\begin{aligned}
X_{n, m}^{ \pm}[\mu]= & V_{n, m}^{ \pm}[\mu] \\
& +\frac{e_{1}}{2}\left((n+m+1) V_{n, m-1}^{ \pm}[\mu]-\frac{1}{n+m+2} V_{n, m+1}^{ \pm}[\mu]\right) \\
& \frac{e_{2}}{2}\left((n+m+1) V_{n, m-1}^{\mp}[\mu]+\frac{1}{n+m+2} V_{n, m+1}^{\mp}[\mu]\right)
\end{aligned}
$$

where the harmonic polynomials $V_{n, m}^{ \pm}[\mu]$ were defined in (8). The $X_{n, m}^{ \pm}[\mu]$ are polynomials in $\mu^{2}$ as well as in $x_{0}, x_{1}, x_{2}$.

Proof. The full operator (15) in spheroidal coordinates (2) is

$$
\begin{gathered}
\partial=\frac{1}{\mu\left(\cosh ^{2} v-\cos ^{2} u\right)}\left[\left(\cos u \sinh v \frac{\partial}{\partial v}-\sin u \cosh v \frac{\partial}{\partial u}\right)\right. \\
-\left(e_{1} \cos \phi+e_{2} \sin \phi\right)\left(\cos u \sinh v \frac{\partial}{\partial u}+\sin u \cosh v \frac{\partial}{\partial v}\right) \\
\left.\times \frac{\left(-e_{1} \sin \phi+e_{2} \cos \phi\right)}{\mu \sin u \sinh v} \frac{\partial}{\partial \phi}\right] .
\end{gathered}
$$

The first line of this expression applied to $U_{n+1, m}^{ \pm}[\mu]$ produces the scalar part of $X_{n, m}^{ \pm}[\mu]$ in (18) and was calculated in [16, 20]. For the nonscalar part, we use the relation (12) to obtain

$$
\begin{gathered}
\frac{2}{\mu^{n+1} \alpha_{n+1, m} \Phi_{m}^{ \pm}}\left(\cos u \sinh v \partial_{u}+\sin u \cosh v \partial_{v}\right) U_{n+1, m}^{ \pm}[\mu] \\
=(n+m+1)(n-m+2)\left(\sin u \cosh v P_{n+1}^{m}(\cos u) P_{n+1}^{m-1}(\cosh v)\right. \\
\left.\quad-\cos u \sinh v P_{n+1}^{m-1}(\cos u) P_{n+1}^{m}(\cosh v)\right) \\
+\left(\sin u \cosh v P_{n+1}^{m}(\cos u) P_{n+1}^{m+1}(\cosh v)\right. \\
\left.\quad+\cos u \sinh v P_{n+1}^{m+1}(\cos u) P_{n+1}^{m}(\cosh v)\right) .
\end{gathered}
$$

Next, the relation

$$
\sqrt{1-t^{2}} P_{n+1}^{m}(t)=(n-m) t P_{n+1}^{m-1}(t)-(n+m) P_{n}^{m-1}(t)
$$

(valid for $|t|<1$, and replacing $1-t^{2}$ with $t^{2}-1$ for $|t|>1$ ) produces

$$
\begin{aligned}
-\frac{\left(\cosh ^{2} v-\cos ^{2} u\right)}{\mu^{n} \alpha_{n+1, m-1}} \widehat{V}_{n, m-1}= & \sin u \cosh v P_{n+1}^{m}(\cos u) P_{n+1}^{m-1}(\cosh v) \\
& -\cos u \sinh v P_{n+1}^{m}(\cosh v) P_{n+1}^{m-1}(\cos u) .
\end{aligned}
$$


Furthermore, using the expression

$$
\left(1-t^{2}\right)^{1 / 2} P_{n+1}^{m}(t)=\frac{1}{2 n+3}\left(P_{n+2}^{m+1}(t)-P_{n}^{m+1}(t)\right),
$$

and its counterpart for $|t|>1$, and then applying (14), we arrive at

$$
\begin{aligned}
\cosh v \sin u P_{n+1}^{m}(\cos u) P_{n+1}^{m+1}(\cosh v) & +\sinh v \cos u P_{n+1}^{m}(\cosh v) P_{n+1}^{m+1}(\cos u) \\
& =\frac{\left(\cosh ^{2} v-\cos ^{2} u\right) \alpha_{n+1, m+1}}{(n-m+1) \mu^{n}} \widehat{V}_{n, m+1} .
\end{aligned}
$$

Similarly, one can prove that

$$
\begin{aligned}
\frac{1}{\sin u \sinh v} & \partial_{\phi} U_{n+1, m}^{ \pm}[\mu]=-\frac{\mu^{n+1} \Phi_{m}^{\mp}}{2 \alpha_{n+1, m}\left(\cosh ^{2} v-\cos ^{2} u\right)} \\
& \times\left[(n+m)(n+m+1)(n-m+2) \widehat{V}_{n, m-1}+\frac{1}{n-m+1} \widehat{V}_{n, m+1}\right] .
\end{aligned}
$$

Combining these three formulas one easily obtains the desired expressions for $\left(\partial / \partial x_{1}\right) U_{n+1, m}^{ \pm}[\mu]$ and $\left(\partial / \partial x_{2}\right) U_{n+1, m}^{ \pm}[\mu]$.

Since the basic spheroidal harmonics of [11] are polynomials of degree $n$, it is clear that the operations of rescaling by $1 / \mu$ or $i / \mu$ and multiplying by $\mu^{n}$ implied in (3) assure that $U_{n, m}^{ \pm}[\mu]$ are polynomials in $\mu$. From the discussion of Section 2 it is clear that $-\mu$ produces the same results as $\mu$, so the only powers of $\mu$ which appear are even.

We will write $\langle\cdot, \cdot\rangle_{[\mu]}=\langle\cdot, \cdot\rangle_{L_{2}\left(\Omega_{\mu}, \mathbb{R}^{3}\right)}$ for the $L_{2}$ inner product in the spheroidal domain $\Omega_{\mu}$, and $\|\cdot\|_{[\mu]}$ for the corresponding norm.

Theorem 3.2. For fixed $\mu$, the monogenic polynomials $X_{n, m}^{ \pm}[\mu]$ are orthogonal with respect to the inner product $\langle\cdot, \cdot\rangle_{[\mu]}$. Their norms are given by

$$
\begin{aligned}
& \left\|X_{n, m}^{ \pm}\right\|_{[\mu]}^{2}=\frac{\pi \mu^{2 n+3}}{(n+2)(n+m+2)(2 n+1) ! !(2 n+3) ! !}[ \\
& \quad(n+2)(n+m)(n+m+1)(n-m+3) !(n+m+2) ! I[n, m-1] \\
& \quad+2 \delta_{0, m}(n+m+2)(n+1) !(n+2) ! I[n, 1] \\
& \quad+(n+2)(n-m+1) !(n+m+2) !(I(n, m+1) \\
& \left.\left.\quad+2(n-m+2)(n+m+1)\left(1+\delta_{0, m}\right) I[n, m]\right)\right]
\end{aligned}
$$


where

$$
I[n, m]=\int_{1}^{1 / \mu} P_{n}^{m}(t) P_{n+2}^{m}(t) d t
$$

Proof. Throughout this proof, we will denote by $[f]_{i}(i=0,1,2)$ the compo-

\begin{tabular}{|c|c|c|}
\hline$n$ & $m$ & $X_{n, m}^{ \pm}$ \\
\hline \multirow[b]{2}{*}{0} & 0 & $X_{0,0}^{+}=1$ \\
\hline & 1 & $\begin{array}{l}X_{0,1}^{+}=e_{1} \\
X_{0,1}^{-}=e_{2}\end{array}$ \\
\hline \multirow{3}{*}{1} & 0 & $X_{1,0}^{+}=2 x_{0}+x_{1} e_{1}+x_{2} e_{2}$ \\
\hline & 1 & $\begin{array}{l}X_{1,1}^{+}=-3 x_{1}+3 x_{0} e_{1} \\
X_{1,1}^{-}=-3 x_{2}+3 x_{0} e_{2}\end{array}$ \\
\hline & 2 & $\begin{array}{l}X_{1,2}^{+}=-6 x_{1} e_{1}+6 x_{2} e_{2} \\
X_{1,2}^{-}=-6 x_{2} e_{1}-6 x_{1} e_{2}\end{array}$ \\
\hline \multirow{4}{*}{2} & 0 & $X_{2,0}^{+}=\left(3 x_{0}^{2}-\frac{3 x_{1}^{2}}{2}-\frac{3 x_{2}^{2}}{2}-\frac{3 \mu^{2}}{5}\right)+3 x_{0} x_{1} e_{1}+3 x_{0} x_{2} e_{2}$ \\
\hline & 1 & $\begin{array}{l}X_{2,1}^{+}=-12 x_{0} x_{1}+\left(6 x_{0}^{2}-\frac{9 x_{1}^{2}}{2}-\frac{3 x_{2}^{2}}{2}-\frac{6 \mu^{2}}{5}\right) e_{1}-3 x_{1} x_{2} e_{2} \\
X_{2,1}^{-}=-12 x_{0} x_{2}-3 x_{1} x_{2} e_{1}+\left(6 x_{0}^{2}-\frac{3 x_{1}^{2}}{2}-\frac{9 x_{2}^{2}}{2}-\frac{6 \mu^{2}}{5}\right) e_{2}\end{array}$ \\
\hline & 2 & $\begin{array}{l}X_{2,2}^{+}=15 x_{1}^{2}-15 x_{2}^{2}-30 x_{0} x_{1} e_{1}+30 x_{0} x_{2} e_{2} \\
X_{2,2}^{-}=30 x_{1} x_{2}-30 x_{0} x_{2} e_{1}-30 x_{0} x_{1} e_{2}\end{array}$ \\
\hline & 3 & $\begin{array}{l}X_{2,3}^{+}=\left(45 x_{1}^{2}-45 x_{2}^{2}\right) e_{1}-90 x_{1} x_{2} e_{2} \\
X_{2,3}^{-}=90 x_{1} x_{2} e_{1}+\left(45 x_{1}^{2}-45 x_{2}^{2}\right) e_{2}\end{array}$ \\
\hline
\end{tabular}

Table 1: Spheroidal monogenic basis polynomials of degree $n=0,1,2$. Observe that the parameter $\mu$ appears when $|n-m| \geq 2$. For each $n$, the last two entries are monogenic constants (and the first entry for $n=0$ ). 
nents of a function $f: \Omega_{\mu} \rightarrow \mathbb{R}^{3}$. Thus

$$
\begin{aligned}
\left\langle X_{n_{1}, m_{1}}^{ \pm}[\mu], X_{n_{2}, m_{2}}^{ \pm}[\mu]\right\rangle_{[\mu]}=\int_{\Omega_{\mu}} & {\left[X_{n_{1}, m_{1}}^{ \pm}[\mu]\right]_{0}\left[X_{n_{2}, m_{2}}^{ \pm}[\mu]\right]_{0} } \\
& +\left[X_{n_{1}, m_{1}}^{ \pm}[\mu]\right]_{1}\left[X_{n_{2}, m_{2}}^{ \pm}[\mu]\right]_{1} \\
& \left.+\left[X_{n_{1}, m_{1}}^{ \pm}[\mu]\right]_{2}\left[X_{n_{2}, m_{2}}^{ \pm}[\mu]\right]_{2}\right) d x
\end{aligned}
$$

\begin{tabular}{|c|c|c|}
\hline$n$ & $m$ & $X_{n, m}^{ \pm}$ \\
\hline \multirow{5}{*}{3} & 0 & $\begin{aligned} X_{3,0}^{+}= & \left(4 x_{0}^{3}-6 x_{0} x_{1}^{2}-6 x_{0} x_{2}^{2}-\frac{12 x_{0} \mu^{2}}{7}\right) \\
& +\left(6 x_{0}^{2} x_{1}-\frac{3 x_{1}^{3}}{2}-\frac{3 x_{1} x_{2}^{2}}{2}-\frac{6 x_{1} \mu^{2}}{7}\right) e_{1} \\
& +\left(6 x_{0}^{2} x_{2}-\frac{3 x_{1}^{2} x_{2}}{2}-\frac{3 x_{2}^{3}}{2}-\frac{6 x_{2} \mu^{2}}{7}\right) e_{2}\end{aligned}$ \\
\hline & 1 & $\begin{aligned} X_{3,1}^{+}= & -30 x_{0}^{2} x_{1}+\frac{15 x_{1}^{3}}{2}+\frac{15 x_{1} x_{2}^{2}}{2}+\frac{30 x_{1} \mu^{2}}{7} \\
& +\left(10 x_{0}^{3}-\frac{45 x_{0} x_{1}^{2}}{2}-\frac{15 x_{0} x_{2}^{2}}{2}-\frac{30 x_{0} \mu^{2}}{7}\right) e_{1}-15 x_{0} x_{1} x_{2} e_{2} \\
X_{3,1}^{-}= & -30 x_{0}^{2} x_{2}+\frac{15 x_{1}^{2} x_{2}}{2}+\frac{15 x_{2}^{3}}{2}+\frac{30 x_{2} \mu^{2}}{7} \\
& -15 x_{0} x_{1} x_{2} e_{1}+\left(5 x_{0}^{3}-\frac{15 x_{0} x_{1}^{2}}{2}-\frac{45 x_{0} x_{2}^{2}}{2}-\frac{30 x_{0} \mu^{2}}{7}\right) e_{2}\end{aligned}$ \\
\hline & 2 & $\begin{aligned} X_{3,2}^{+}= & 90 x_{0} x_{1}^{2}-90 x_{0} x_{2}^{2}+\left(-90 x_{0}^{2} x_{1}+30 x_{1}^{3}+\frac{90 x_{1} \mu^{2}}{7}\right) e_{1} \\
& +\left(90 x_{0}^{2} x_{2}-30 x_{2}^{3}-\frac{90 x_{2} \mu^{2}}{7}\right) e_{2} \\
X_{3,2}^{-}= & 180 x_{0} x_{1} x_{2}+\left(-90 x_{0}^{2} x_{2}+45 x_{1}^{2} x_{2}+15 x_{2}^{3}+\frac{90 x_{2} \mu^{2}}{7}\right) e_{1} \\
& +\left(-90 x_{0}^{2} x_{1}+15 x_{1}^{3}+45 x_{1} x_{2}^{2}+\frac{90 x_{1} \mu^{2}}{7}\right) e_{2}\end{aligned}$ \\
\hline & 3 & $\begin{array}{l}X_{3,3}^{+}=-105 x_{1}^{3}+315 x_{1} x_{2}^{2}+\left(315 x_{0} x_{1}^{2}-315 x_{0} x_{2}^{2}\right) e_{1}-630 x_{0} x_{1} x_{2} e_{2} \\
X_{3,3}^{-}=-315 x_{1}^{2} x_{2}+105 x_{2}^{3}+630 x_{0} x_{1} x_{2} e_{1}+\left(315 x_{0} x_{1}^{2}-315 x_{0} x_{2}^{2}\right) e_{2}\end{array}$ \\
\hline & 4 & $\begin{array}{l}X_{3,4}^{+}=\left(-420 x_{1}^{3}+1260 x_{1} x_{2}^{2}\right) e_{1}+\left(1260 x_{1}^{2} x_{2}-420 x_{2}^{3}\right) e_{2} \\
X_{3,4}^{-}=\left(-1260 x_{1}^{2} x_{2}+420 x_{2}^{3}\right) e_{1}+\left(-420 x_{1}^{3}+1260 x_{1} x_{2}^{2}\right) e_{2}\end{array}$ \\
\hline
\end{tabular}

Table 2: Spheroidal monogenic polynomials of degree $n=3$. 
where $d x=d x_{0} d x_{1} d x_{2}$. By Theorem 3.1 and Proposition 2.1,

$$
\int_{\Omega_{\mu}}\left[X_{n_{1}, m_{1}}^{ \pm}[\mu]\right]_{0}\left[X_{n_{2}, m_{2}}^{ \pm}[\mu]\right]_{0} d x=\left\|V_{n_{1}, m_{1}}^{ \pm}[\mu]\right\|_{[\mu]}^{2} \delta_{n_{1}, n_{2}} \delta_{m_{1}, m_{2}} .
$$

Thus, to verify the orthogonality of the $X_{n, m}^{ \pm}[\mu]$ it suffices to show that the vector parts of the functions $X_{n, m}^{ \pm}[\mu]$ are orthogonal.

Expanding the integrands and applying the trigonometric identities

$$
\begin{aligned}
\Phi_{m_{1}-1}^{ \pm} \Phi_{m_{2}-1}^{ \pm}+\Phi_{m_{1}-1}^{\mp} \Phi_{m_{2}-1}^{\mp} & =\Phi_{m_{1}-m_{2}}^{+}, \\
\Phi_{m_{1}+1}^{ \pm} \Phi_{m_{2}+1}^{ \pm}+\Phi_{m_{1}+1}^{\mp} \Phi_{m_{2}+1}^{\mp} & =\Phi_{m_{1}-m_{2}}^{+}, \\
-\Phi_{m_{1}-1}^{ \pm} \Phi_{m_{2}+1}^{ \pm}+\Phi_{m_{1}-1}^{\mp} \Phi_{m_{2}+1}^{\mp} & =\mp \Phi_{m_{1}+m_{2}}^{+} \\
-\Phi_{m_{1}+1}^{ \pm} \Phi_{m_{2}-1}^{ \pm}+\Phi_{m_{1}+1}^{\mp} \Phi_{m_{2}-1}^{\mp} & =\mp \Phi_{m_{1}+m_{2}}^{+}
\end{aligned}
$$

we obtain that

$$
\begin{aligned}
\int_{\Omega_{\mu}} & \left(\left[X_{n_{1}, m_{1}}^{ \pm}[\mu]\right]_{1}\left[X_{n_{2}, m_{2}}^{ \pm}[\mu]\right]_{1}+\left[X_{n_{1}, m_{1}}^{ \pm}[\mu]\right]_{2}\left[X_{n_{2}, m_{2}}^{ \pm}[\mu]\right]_{2}\right) d x \\
= & \frac{1}{4}\left(p_{1} p_{2} \int_{\Omega_{\mu}} \widehat{V}_{n_{1}, m_{1}-1}[\mu] \widehat{V}_{n_{2}, m_{2}-1}[\mu] \Phi_{m_{1}-m_{2}}^{+} d x\right. \\
& \mp \frac{p_{1}}{p_{2}+1} \int_{\Omega_{\mu}} \widehat{V}_{n_{1}, m_{1}-1}[\mu] \widehat{V}_{n_{2}, m_{2}+1}[\mu] \Phi_{m_{1}+m_{2}}^{+} d x \\
& \mp \frac{p_{2}}{p_{1}+1} \int_{\Omega_{\mu}} \widehat{V}_{n_{1}, m_{1}+1}[\mu] \widehat{V}_{n_{2}, m_{2}-1}[\mu] \Phi_{m_{1}+m_{2}}^{+} d x \\
& \left.+\frac{1}{\left(p_{1}+1\right)\left(p_{2}+1\right)} \int_{\Omega_{\mu}} \widehat{V}_{n_{1}, m_{1}+1}[\mu] \widehat{V}_{n_{2}, m_{2}+1}[\mu] \Phi_{m_{1}-m_{2}}^{+} d x\right)
\end{aligned}
$$

where $p_{i}=m_{i}+n_{i}+1(i=1,2)$. We continue the calculation only for the prolate case, applying the coordinates (2) which give

$$
d x=d R d \phi
$$

where $d R=\mu^{3}\left(\cosh ^{2} v-\cos ^{2} u\right) \sin u \sinh v d u d v$. 
The identities $\int_{0}^{2 \pi} \Phi_{m_{1} \pm m_{2}}(\phi) d \phi=2 \pi \delta_{m_{1}, m_{2}}$ for $m_{1}, m_{2}>0$ imply that

$$
\begin{aligned}
\int_{\Omega_{\mu}} & \left(\left[X_{n_{1}, m_{1}}^{ \pm}[\mu]\right]_{1}\left[X_{n_{2}, m_{2}}^{ \pm}[\mu]\right]_{1}+\left[X_{n_{1}, m_{1}}^{ \pm}[\mu]\right]_{2}\left[X_{n_{2}, m_{2}}^{ \pm}[\mu]\right]_{2}\right) d x \\
= & \frac{\pi p_{1}\left(n_{2}+m_{1}+1\right) \delta_{m_{1}, m_{2}}}{2} \int_{0}^{1 / \mu} \int_{0}^{\pi} \widehat{V}_{n_{1}, m_{1}-1}[\mu] \widehat{V}_{n_{2}, m_{1}-1}[\mu] d R \\
& \mp \pi \frac{n_{2}+1}{2\left(n_{1}+2\right)} \delta_{m_{1}, 0} \int_{0}^{1 / \mu} \int_{0}^{\pi} \widehat{V}_{n_{1}, 1}[\mu] \widehat{V}_{n_{2},-1}[\mu] d R \\
& \mp \pi \frac{n_{1}+1}{2\left(n_{2}+2\right)} \delta_{m_{1}, 0} \int_{0}^{1 / \mu} \int_{0}^{\pi} \widehat{V}_{n_{1},-1}[\mu] \widehat{V}_{n_{2}, 1}[\mu] d R \\
& +\frac{\pi}{2\left(p_{1}+1\right)\left(n_{2}+m_{1}+1\right)} \delta_{m_{1}, m_{2}} \int_{0}^{1 / \mu} \int_{0}^{\pi} \widehat{V}_{n_{1}, m_{1}+1}[\mu] \widehat{V}_{n_{2}, m_{1}+1}[\mu] d R .
\end{aligned}
$$

In consequence, using (17), we have

$$
\begin{gathered}
\int_{\Omega_{\mu}}\left(\left[X_{n_{1}, m_{1}}^{ \pm}[\mu]\right]_{1}\left[X_{n_{2}, m_{2}}^{ \pm}[\mu]\right]_{1}+\left[X_{n_{1}, m_{1}}^{ \pm}[\mu]\right]_{2}\left[X_{n_{2}, m_{2}}^{ \pm}[\mu]\right]_{2}\right) d x \\
=\frac{\pi p_{1}\left(n_{2}+m_{1}+1\right) \delta_{m_{1}, m_{2}}}{2} \int_{0}^{1 / \mu} \int_{0}^{\pi} \widehat{V}_{n_{1}, m_{1}-1}[\mu] \widehat{V}_{n_{2}, m_{1}-1}[\mu] d R \\
\quad \pm \frac{\pi}{\left(n_{1}+2\right)\left(n_{2}+2\right)} \delta_{m_{1}, 0} \int_{0}^{1 / \mu} \int_{0}^{\pi} \widehat{V}_{n_{1}, 1}[\mu] \widehat{V}_{n_{2}, 1}[\mu] d R \\
\quad+\frac{\pi}{2 p_{1}\left(n_{2}+m_{1}+1\right)} \delta_{m_{1}, m_{2}} \int_{0}^{1 / \mu} \int_{0}^{\pi} \widehat{V}_{n_{1}, m_{1}+1}[\mu] \widehat{V}_{n_{2}, m_{1}+1}[\mu] d R
\end{gathered}
$$

Using Proposition 2.3, and applying again the orthogonality of Theorem 2.1, we are left with

$$
\begin{aligned}
\int_{\Omega_{\mu}}\left(\left[X_{n_{1}, m_{1}}^{ \pm}[\mu]\right]_{1}\left[X_{n_{2}, m_{2}}^{ \pm}[\mu]\right]_{1}+\left[X_{n_{1}, m_{1}}^{ \pm}[\mu]\right]_{2}\left[X_{n_{2}, m_{2}}^{ \pm}[\mu]\right]_{2}\right) d x \\
=\frac{\pi \mu^{2 n_{1}+3}}{\left(n_{1}+2\right)\left(2 n_{1}+1\right) ! !\left(2 n_{1}+3\right) ! !}\left[\left(n_{1}+2\right)\left(n_{1}+m_{1}+1\right) !\right. \\
\quad \times\left(\left(n_{1}+m_{1}\right)\left(n_{1}+m_{1}+1\right)\left(n_{1}-m_{1}+3\right) ! I\left[n_{1}, m_{1}-1\right]\right. \\
\left.\quad+\left(n_{1}-m_{1}+1\right) ! I\left[n_{1}, m_{1}+1\right]\right) \\
\left.\quad+2\left(n_{1}+1\right) !\left(n_{1}+2\right) ! I\left[n_{1}, 1\right] \delta_{0, m}\right] \delta_{m_{1}, m_{2}} \delta_{n_{1}, n_{2}}
\end{aligned}
$$


with $I[n, m]$ defined in (22) . Combining (23) and (24), we conclude that

$$
\begin{aligned}
& \left\langle X_{n_{1}, m_{1}}^{+}, X_{n_{2}, m_{2}}^{+}\right\rangle_{[\mu]}=0 \text { when } n_{1} \neq n_{2} \text { or } m_{1} \neq m_{2}, \\
& \left\langle X_{n_{1}, m_{1}}^{-}, X_{n_{2}, m_{2}}^{-}\right\rangle_{[\mu]}=0 \text { when } n_{1} \neq n_{2} \text { or } m_{1} \neq m_{2} .
\end{aligned}
$$

Using once more the orthogonality of the system $\left\{\Phi_{m}^{ \pm}\right\}$on $[0,2 \pi]$, we conclude that

$$
\left\langle X_{n_{1}, m_{1}}^{ \pm}, X_{n_{2}, m_{2}}^{\mp}\right\rangle_{[\mu]}=0
$$

when the indices do not coincide. The calculation of the norms comes from taking $n_{1}=n_{2}$ and $m_{1}=m_{2}$ in (24) and adding the expression (9).

In the next subsection we make precise how the monogenic polynomials sit in the space of harmonic polynomials. It is well known (cf. [14]) that the dimension of the space $\mathcal{M}^{(n)}$ of homogeneous monogenic polynomials of degree $n$ in $\left(x_{0}, x_{1}, x_{2}\right)$ is $2 n+3$ (this does not depend on the domain $\Omega$ ). Since the polynomials we are working with are not homogeneous, we consider the space $\mathcal{M}_{*}^{(n)}=\bigcup_{0<k<n} \mathcal{M}^{(n)}$ of monogenic polynomials of degree $n$, a class which is not altered by adding monogenic polynomials of lower degree. Thus

$$
\operatorname{dim} \mathcal{M}_{*}^{(n)}=\sum_{k=0}^{n}(2 k+3)=(n+3)(n+1) .
$$

Consider the collections of $2 n+3$ polynomials

$$
B_{n}[\mu]=\left\{X_{k, m}^{+}[\mu], 0 \leq m \leq k+1\right\} \cup\left\{X_{k, m}^{-}[\mu], 1 \leq m \leq k+1\right\} .
$$

By Theorem 3.2 and (25), the union

$$
\bigcup_{0 \leq k \leq n} B_{n}[\mu]
$$

is an orthogonal basis for $\mathcal{M}_{*}^{(n)}$. By the symmetric form taken by $X_{m, n}^{ \pm}[\mu]$ in (18), we know that when $m \neq 0$,

$$
\left\|X_{n, m}^{+}[\mu]\right\|_{[\mu]}=\left\|X_{n, m}^{-}[\mu]\right\|_{[\mu]} .
$$




\subsection{Spheroidal ambigenic polynomials}

Facts about antimonogenic functions are generally trivial modifications of facts about monogenic functions, obtained by taking the conjugate. However, in order to discuss contragenic functions below it will be necessary to discuss first the subspace of the $\mathbb{R}^{3}$-valued harmonic functions generated by the monogenic and antimonogenic functions together. Elements of this space were termed ambigenic functions in [1].

It is known [17], and easy to verify, that $f$ is antimonogenic if and only if $\bar{f}$ is monogenic. The decomposition of an ambigenic function as a sum of a monogenic and an antimonogenic function is not unique, so we must take into account the set $\mathcal{M}(\Omega) \cap \overline{\mathcal{M}}(\Omega)$ of monogenic constants in the domain $\Omega \subseteq \mathbb{R}^{3}$. Monogenic constants do not depend on $x_{0}$ and can be expressed as

$$
f=a_{0}+f_{1} e_{1}+f_{2} e_{2}
$$

where $a_{0} \in \mathbb{R}$ is a constant, and $f_{1}-i f_{2}$ is an ordinary holomorphic function of the complex variable $x_{1}+i x_{2}$. There are natural projections of $\mathcal{M}(\Omega)$ onto the subspaces

$$
\begin{aligned}
\operatorname{Sc} \mathcal{M}(\Omega) & =\{\operatorname{Sc} f \mid f \in \mathcal{M}(\Omega)\} \subseteq \operatorname{Har}_{\mathbb{R}}(\Omega) \\
\operatorname{Vec} \mathcal{M}(\Omega) & =\{\operatorname{Vec} f \mid f \in \mathcal{M}(\Omega)\} \subseteq \operatorname{Har}_{\{0\} \oplus \mathbb{R}^{2}}(\Omega),
\end{aligned}
$$

where $\operatorname{Har}_{\mathbb{R}}(\Omega)$ denotes the space of real-valued harmonic functions defined in $\Omega$. Note that $\operatorname{Sc} \mathcal{M}(\Omega)=\operatorname{Sc} \overline{\mathcal{M}}(\Omega)$ and $\operatorname{Vec} \mathcal{M}(\Omega)=-\operatorname{Vec} \overline{\mathcal{M}}(\Omega)$. When $\Omega$ is simply-connected, $\operatorname{Sc} \mathcal{M}(\Omega)=\operatorname{Har}_{\mathbb{R}}(\Omega)$.

It is known [1], that the real dimension of the space $\mathcal{M}^{(n)}+\overline{\mathcal{M}}^{(n)}$ of homogeneous ambigenic polynomials is $4 n+4$ when $n \geq 1$. As discussed in the previous section, the basis polynomials for spheroidal functions are not homogenous. The dimension of the space $\mathcal{M}_{*}^{(n)}+\overline{\mathcal{M}}_{*}^{(n)}$ of ambigenic polynomials of degree at most $n$ is

$$
\begin{aligned}
\operatorname{dim}\left(\mathcal{M}_{*}^{(n)}+\overline{\mathcal{M}}_{*}^{(n)}\right) & =\sum_{k=0}^{n} \operatorname{dim}\left(\mathcal{M}^{(k)}+\overline{\mathcal{M}}^{(k)}\right) \\
& =3+\sum_{k=1}^{n}(4 k+4)=2 n(n+3)+3 .
\end{aligned}
$$

Observe that we have by (18) that

$$
X_{n, n+1}^{ \pm}[\mu]=(n+1)\left(V_{n, n}^{ \pm}[\mu] e_{1} \mp V_{n, n}^{\mp}[\mu] e_{2}\right),
$$


and

$$
V_{n, n}^{\mp}[\mu]=(-1)^{n}(2 n+1) ! !\left(x_{1}^{2}+x_{2}^{2}\right)^{n / 2} .
$$

The first equation shows that $X_{n, n+1}^{ \pm}[\mu]$ has vanishing scalar part; i.e. they are the negatives of their conjugates. Consequently, the $X_{n, n+1}^{ \pm}[\mu]$ are monogenic constants. This observation makes it possible to give a basis for the ambigenic polynomials defined in spheroidal domains. In the following we take into account the fact that

Lemma 3.3. For $m \neq 0$,

$$
\left\langle X_{k, m}^{+}[\mu], \bar{X}_{k, m}^{+}[\mu]\right\rangle_{[\mu]}=\left\langle X_{k, m}^{-}[\mu], \bar{X}_{k, m}^{-}[\mu]\right\rangle_{[\mu]} .
$$

Proof. Indeed,

$$
\begin{aligned}
\left\langle X_{k, m}^{+}[\mu], \bar{X}_{k, m}^{+}[\mu]\right\rangle_{[\mu]}=\int_{\Omega_{\mu}}\left(\left[X_{k, m}^{+}[\mu]\right]_{0}^{2}-\left[X_{k, m}^{+}[\mu]\right]_{1}^{2}-\left[X_{k, m}^{+}[\mu]\right]_{2}^{2}\right) d x \\
=\int_{0}^{\pi} \int_{0}^{1 / \mu}\left(\widehat{V}_{k, m}[\mu]\right)^{2} d v d u \int_{0}^{2 \pi} \cos ^{2} m \phi d \phi \\
\quad-\frac{1}{4} \int_{0}^{\pi} \int_{0}^{1 / \mu}\left((k+m+1) \widehat{V}_{k, m-1}[\mu]-\frac{1}{k+m+2} \widehat{V}_{k, m+1}[\mu]\right)^{2} d v d u \\
\quad \times \int_{0}^{2 \pi} \cos ^{2} m \phi d \phi \\
\quad-\frac{1}{4} \int_{0}^{\pi} \int_{0}^{1 / \mu}\left((k+m+1) \widehat{V}_{k, m-1}[\mu]-\frac{1}{k+m+2} \widehat{V}_{k, m+1}[\mu]\right)^{2} d v d u \\
\quad \times \int_{0}^{2 \pi} \sin ^{2}(m \phi) d \phi .
\end{aligned}
$$

Since $m \neq 0$, the two values $\int_{0}^{2 \pi} \Phi_{m}^{ \pm}(\phi)^{2} d \phi$ are equal, and therefore

$$
\begin{aligned}
\left\langle X_{k, m}^{+}[\mu], \bar{X}_{k, m}^{+}[\mu]\right\rangle_{[\mu]} & =\int_{\Omega_{\mu}}\left(\left[X_{k, m}^{-}[\mu]\right]_{0}^{2}-\left[X_{k, m}^{-}[\mu]\right]_{1}^{2}-\left[X_{k, m}^{-}[\mu]\right]_{2}^{2}\right) d x \\
& =\left\langle X_{k, m}^{-}[\mu], \bar{X}_{k, m}^{-}[\mu]\right\rangle_{[\mu]} .
\end{aligned}
$$

It is not possible to extract from the list $\left\{X_{n, m}^{ \pm}, \bar{X}_{n, m}^{ \pm}\right\}$an orthogonal basis of ambigenic functions, but only a small modification is necessary. Define the 
functions

$$
\begin{aligned}
Y_{n, m}^{++}[\mu] & =X_{n, m}^{+}[\mu], \\
Y_{n, m}^{-+}[\mu] & =X_{n, m}^{-}[\mu], \\
Y_{n, m}^{+-}[\mu] & =\bar{X}_{n, m}^{+}[\mu]-\gamma_{n, m}[\mu] X_{n, m}^{+}[\mu], \\
Y_{n, m}^{--}[\mu] & =\bar{X}_{n, m}^{-}[\mu]-\gamma_{n, m}[\mu] X_{n, m}^{-}[\mu],
\end{aligned}
$$

where

$$
\gamma_{n, m}[\mu]=\left\{\begin{array}{cl}
\frac{\left\langle X_{n, m}^{+}[\mu], \bar{X}_{n, m}^{+}[\mu]\right\rangle_{[\mu]}}{\left\|X_{n, m}^{+}[\mu]\right\|_{[\mu]}^{2}}, & \text { if } 0 \leq m \leq n \\
0, & \text { if } m=n+1
\end{array}\right.
$$

and

$$
\begin{aligned}
& Y_{0, m}^{++}[\mu]=X_{0, m}^{+}[\mu] \text { for } m=0,1, \\
& Y_{0,1}^{-+}[\mu]=X_{0,1}^{-}[\mu] .
\end{aligned}
$$

Proposition 3.4. The collection of $2 n(n+3)+3$ polynomials

$$
\begin{aligned}
& \left\{Y_{k, m}^{++}: 0 \leq m \leq k+1\right\} \cup\left\{Y_{k, m}^{-+}: 0 \leq m \leq k\right\} \\
& \cup\left\{Y_{k, m}^{+-}: 0 \leq m \leq k\right\} \cup\left\{Y_{k, m}^{--}: 0 \leq m \leq k+1\right\},
\end{aligned}
$$

$0 \leq k \leq n$, is an orthogonal basis in $L_{2}\left(\Omega_{\mu}\right)$ for the subspace of ambigenic polynomials of degree at most $n$.

Proof. Throughout this proof, in view of the fact that $\mu$ is fixed, we simply write $X_{k, m}^{ \pm}, Y_{k, m}^{ \pm \pm}, \gamma_{k, m}$ for $X_{k, m}^{ \pm}[\mu], Y_{k, m}^{ \pm, \pm}[\mu], \gamma_{k, m}[\mu]$. Since there are $2 n(n+3)+3$ ambigenic functions in the given list, it suffices to prove the orthogonality to conclude that they generate the ambigenic polynomials. Because the set

$$
\left\{X_{k, 0}^{+}, X_{k, m}^{+}, X_{k, m}^{-} \mid k=0, \ldots, n, m=1, \ldots, k+1\right\}
$$

is an orthogonal basis of $\mathcal{M}_{*}^{(n)}$ in $\Omega_{\mu}$, it follows at once that

$$
\left\langle Y_{k, m}^{++}, \overline{Y_{k, m}^{-+}}\right\rangle_{[\mu]}=\left\langle Y_{k, m}^{++}, \overline{Y_{k, m}^{--}}\right\rangle_{[\mu]}=\left\langle Y_{k, m}^{+-}, \overline{Y_{k, m}^{-+}}\right\rangle_{[\mu]}=\left\langle Y_{k, m}^{+-}, \overline{Y_{k, m}^{--}}\right\rangle_{[\mu]}=0 .
$$

Since

$$
\begin{aligned}
\left\langle Y_{k_{1}, m_{1}}^{+-}, Y_{k_{2}, m_{2}}^{+-}\right\rangle_{[\mu]}= & \left\langle\bar{X}_{k_{1}, m_{1}}^{+}-\gamma_{k_{1}, m_{1}} X_{k_{1}, m_{1}}^{+}, \bar{X}_{k_{2}, m_{2}}^{+}-\gamma_{k_{2}, m_{2}} X_{k_{2}, m_{2}}^{+}\right\rangle_{[\mu]} \\
= & \left\langle\bar{X}_{k_{1}, m_{1}}^{+}, \bar{X}_{k_{2}, m_{2}}^{+}\right\rangle_{[\mu]}-\gamma_{k_{2}, m_{2}}\left\langle\bar{X}_{k_{1}, m_{1}}^{+}, X_{k_{2}, m_{2}}^{+}\right\rangle_{[\mu]} \\
& -\gamma_{k_{1}, m_{1}}\left\langle X_{k_{1}, m_{1}}^{+}, \bar{X}_{k_{2}, m_{2}}^{+}\right\rangle_{[\mu]} \\
& +\gamma_{k_{1}, m_{1}} \gamma_{k_{2}, m_{2}}\left\langle X_{k_{1}, m_{1}}^{+}, X_{k_{2}, m_{2}}^{+}\right\rangle_{[\mu]},
\end{aligned}
$$


it will be enough to study $\left\langle\bar{X}_{k_{1}, m_{1}}^{+}, X_{k_{2}, m_{2}}^{+}\right\rangle_{[\mu]}$ and $\left\langle X_{k_{1}, m_{1}}^{+}, \bar{X}_{k_{2}, m_{2}}^{+}\right\rangle_{[\mu]}$ :

$$
\begin{aligned}
\left\langle\bar{X}_{k_{1}, m_{1}}^{+}, X_{k_{2}, m_{2}}^{+}\right\rangle_{[\mu]}= & \int_{\Omega_{\mu}}\left(\left[X_{k_{1}, m_{1}}^{+}\right]_{0}\left[X_{k_{2}, m_{2}}^{+}\right]_{0}\right. \\
& \left.-\left(\left[X_{k_{1}, m_{1}}^{+}\right]_{1}\left[X_{k_{2}, m_{2}}^{+}\right]_{1}+\left[X_{k_{1}, m_{1}}^{+}\right]_{2}\left[X_{k_{2}, m_{2}}^{+}\right]_{2}\right)\right) d x,
\end{aligned}
$$

but from the proof of Proposition 3.2, we obtain that

$$
\left\langle\bar{X}_{k_{1}, m_{1}}^{+}, X_{k_{2}, m_{2}}^{+}\right\rangle_{[\mu]}=\left(\left\|\operatorname{Sc} X_{k_{1}, m_{1}}^{+}\right\|_{[\mu]}^{2}-\left\|\operatorname{Vec} X_{k_{1}, m_{1}}^{+}\right\|_{[\mu]}^{2}\right) \delta_{k_{1}, k_{2}} \delta_{m_{1}, m_{2}} .
$$

Now we note that

$$
\left\langle Y_{k_{1}, m_{1}}^{++}, Y_{k_{2}, m_{2}}^{+-}\right\rangle_{[\mu]}=\left\langle X_{k_{1}, m_{1}}^{+}, \bar{X}_{k_{2}, m_{2}}^{+}-\gamma_{k_{2}, m_{2}} X_{k_{2}, m_{2}}^{+}\right\rangle_{[\mu]} .
$$

By the above observations, these functions are orthogonal when $k_{1} \neq k_{2}$ or $m_{1} \neq m_{2}$, and when the indices coincide,

$$
\left\langle Y_{k, m}^{++}, Y_{k, m}^{+-}\right\rangle_{[\mu]}=\left\langle X_{k, m}^{+}, \bar{X}_{k, m}^{+}\right\rangle_{[\mu]}-\frac{\left\langle X_{k, m}^{+}, \bar{X}_{k, m}^{+}\right\rangle_{[\mu]}}{\left\|X_{k, m}^{+}\right\|_{[\mu]}^{2}}\left\langle X_{k, m}^{+}, X_{k, m}^{+}\right\rangle_{[\mu]}=0 .
$$

Moreover, by the orthogonality of the system $\left\{\Phi_{k}^{+}, \Phi_{l}^{-} \mid k \geq 0, l>0\right\}$, it is clear that $\left\langle Y_{k_{1}, m_{1}}^{++}, Y_{k_{2}, m_{2}}^{--}\right\rangle_{[\mu]}=0$, and further $\left\langle Y_{k, m}^{++}, Y_{k, m}^{--}\right\rangle_{[\mu]}=0$. Finally,

$$
\left\langle Y_{k, m}^{-+}, Y_{k, m}^{--}\right\rangle_{[\mu]}=\left\langle X_{k, m}^{-}, \bar{X}_{k, m}^{-}\right\rangle_{[\mu]}-\frac{\left\langle X_{k, m}^{+}, \bar{X}_{k, m}^{+}\right\rangle_{[\mu]}}{\left\|X_{k, m}^{+}\right\|_{[\mu]}^{2}}\left\|X_{k, m}^{-}\right\|_{[\mu]}^{2} .
$$

Note that

$$
\left\langle X_{k, m}^{-}, \bar{X}_{k, m}^{-}\right\rangle_{[\mu]}=\left\langle X_{k, m}^{+}, \bar{X}_{k, m}^{+}\right\rangle_{[\mu]}
$$

and $\left\|X_{k, m}^{-}\right\|_{[\mu]}^{2}=\left\|X_{k, m}^{+}\right\|_{[\mu]}^{2}$, when $m \neq 0$. Therefore $\left\langle Y_{k, m}^{-+}, Y_{k, m}^{--}\right\rangle_{[\mu]}=0$.

It can shown, with more work, that $X_{n, m}^{ \pm}$are (up to rescaling) the same polynomials defined in [16] (cf. [26]); we will not need this fact here.

\section{Spheroidal contragenic functions}

We now come to our main subject. It is well known that every $\mathbb{C}$-valued harmonic function in a simply connected domain in the complex plane $\mathbb{C}$ is expressible as the sum of a holomorphic function and an antiholomorphic function; these two elements are unique up to a constant summand. There 
are many generalizations of this fact for monogenic functions on quaternions [30] and Clifford algebras [2]. A similar result for monogenic functions from $\mathbb{R}^{3} \rightarrow \mathbb{H}$ is given in [7]. However, it was discovered in [1] by a dimension count that the corresponding statement for monogenic functions $\mathbb{R}^{3} \rightarrow \mathbb{R}^{3}$ does not hold, due to the fact that the multiplication in $\mathbb{R}^{3}$ is not a closed operation in $\mathbb{H}$. In other words, there are harmonic functions which are not expressible as the sum of a monogenic and an antimonogenic function.

We summarize the dimensions over $\mathbb{R}$ of the relevant spaces of polynomials in Table 3, The subscript $*$ refers to polynomials of degree at most $n$.

\begin{tabular}{|c|c|}
\hline Space of polynomials & $\operatorname{dim}_{\mathbb{R}}$ \\
\hline $\operatorname{Har}_{*}^{(n)}(\mathbb{R})$ & $(n+1)^{2}$ \\
\hline $\operatorname{Har}_{*}^{(n)}\left(\mathbb{R}^{3}\right)$ & $3(n+1)^{2}$ \\
\hline $\mathcal{M}_{*}^{(n)}, \overline{\mathcal{M}}_{*}^{(n)}$ & $(n+3)(n+1)$ \\
\hline $\mathcal{M}_{*}^{(n)} \cap \overline{\mathcal{M}}_{*}^{(n)}$ & $2 n+3$ \\
\hline $\mathcal{M}_{*}^{(n)}+\overline{\mathcal{M}}_{*}^{(n)}$ & $2\left(n^{2}+3 n+1\right)+1$ \\
\hline
\end{tabular}

Table 3: Dimensions of spaces of polynomials $(n \geq 0)$.

\subsection{Spheroidal contragenic polynomials}

One way to quantify the failure of a harmonic function to be ambigenic is via orthogonal complements. We will write

$$
\mathcal{M}_{2}(\Omega)=\mathcal{M}(\Omega) \cap L_{2}(\Omega) .
$$

Since scalar-valued (i.e. $\mathbb{R} e_{0}$-valued) functions are by definition orthogonal in $L_{2}(\Omega)$ to functions which take values in $\mathbb{R} e_{1}+\mathbb{R} e_{2}$, there is a natural orthogonal direct sum decomposition of the space of square-integrable ambigenic functions, namely

$$
\mathcal{M}_{2}(\Omega)+\overline{\mathcal{M}}_{2}(\Omega)=\operatorname{Sc} \mathcal{M}_{2}(\Omega) \oplus \operatorname{Vec} \mathcal{M}_{2}(\Omega) .
$$

In any domain $\Omega$, a harmonic function $h \in \operatorname{Har}(\Omega) \cap L_{2}(\Omega)$ is called $\Omega$-contragenic when it is orthogonal to all square-integrable ambigenic functions, that is, if it lies in

$$
\mathcal{N}(\Omega)=\left(\mathcal{M}_{2}(\Omega)+\overline{\mathcal{M}}_{2}(\Omega)\right)^{\perp},
$$


where the orthogonal complement is taken in $\operatorname{Har}(\Omega) \cap L_{2}(\Omega)$. Let $\mathcal{N}^{(n)}(\Omega) \subset$ $\mathcal{N}(\Omega)$ denote the subspace of contragenic polynomials of degree $n$, and let $\mathcal{N}_{*}^{(n)}(\Omega) \subset \mathcal{N}(\Omega)$ be the subspace of polynomials of degree $\leq n$. Unlike the spaces of harmonic, monogenic, antimonogenic and ambigenic polynomials, the definition of $\mathcal{N}^{(n)}(\Omega)$ and $\mathcal{N}_{*}^{(n)}(\Omega)$ involves the $L_{2}$ inner product and thus depends on $\Omega$.

We now return to spheroids, and write $\mathcal{N}_{*}^{(n)}[\mu]=\mathcal{N}_{*}^{(n)}\left(\Omega_{\mu}\right)$. Let $n \geq 1$. In [1], it was proved that for $n \geq 1$, the homogeneous polynomials of degree $n$ which are contragenic on the sphere $\Omega_{0}$ form a space of dimension $2 n-1$. It was also observed when an $\mathbb{R}$-valued harmonic homogeneous polynomial is completed as the scalar part of a monogenic function (unique up to adding a monogenic constant), the vector part can also be taken to be a homogeneous polynomial of the same degree.

Since the spheroidal harmonics and monogenics are not homogeneous, it is preferable to combine the dimensions up to $n$; for the sphere we have $\operatorname{dim} \mathcal{N}_{*}^{(n)}[0]=n^{2}$. Since the dimension of an orthogonal complement within a fixed vector space does not depend on the inner product used, and since the harmonic and the ambigenic polynomials of degree $\leq n$ do not depend on the domain, it is clear that we have in general

$$
\operatorname{dim} \mathcal{N}_{*}^{(n)}[\mu]=n^{2} .
$$

We now give an explicit construction of a basis of the $\mathcal{N}_{*}^{(n)}$, using as building blocks the components of the monogenic functions. Write

$$
a_{n, m}[\mu]=\frac{\left\|V_{n, m+1}^{+}[\mu]\right\|_{[\mu]}^{2}}{(n+m+1)(n+m+2)^{2}\left\|V_{n, m-1}^{+}[\mu]\right\|_{[\mu]}^{2}} .
$$

Definition 4.1. Let $n \geq 1$. The basic contragenic polynomials for $\Omega_{\mu}$ are

$$
\begin{aligned}
Z_{n, m}^{ \pm}[\mu]= & \left.\frac{a_{n, m}}{n+m+1}\left(\operatorname{Vec} X_{n, m}^{\mp}[\mu] \mp X_{n, m}^{ \pm}[\mu] e_{3}\right) \pm\left[X_{n, m}^{ \pm}[\mu]\right]_{0} e_{3}\right] \\
& +\left(-\operatorname{Vec} X_{n, m}^{\mp}[\mu] \mp X_{n, m}^{ \pm}[\mu] e_{3} \pm\left[X_{n, m}^{ \pm}[\mu]\right]_{0} e_{3}\right)
\end{aligned}
$$

for $1 \leq m \leq n-1$, while for $m=0$ we define

$$
Z_{n, 0}[\mu]=\frac{1}{n+2}\left(V_{n, 1}^{-}[\mu] e_{1}-V_{n, 1}^{+}[\mu] e_{2}\right) .
$$


Examples are given in Table 4. In what follows, we will continue to write $X_{n, m}^{ \pm}, Z_{n, m}^{ \pm}, a_{n, m}$ in place of $X_{n, m}^{ \pm}[\mu], Z_{n, m}^{ \pm}[\mu], a_{n, m}[\mu]$ when $\mu$ is fixed. The basic contragenic polynomials may be expressed in terms of their $e_{1}, e_{2}$ components by defining

$$
b_{n, m}^{ \pm}=\frac{a_{n, m} \pm(n+m+1)}{n+m+1}
$$

then

$$
\begin{aligned}
Z_{n, m}^{ \pm}=\left(b_{n, m}^{-}\left[X_{n, m}^{\mp}\right]_{1} \mp b_{n, m}^{+}\left[X_{n, m}^{ \pm}\right]_{2}\right) e_{1} \\
+\left(b_{n, m}^{-}\left[X_{n, m}^{\mp}\right]_{2} \pm b_{n, m}^{+}\left[X_{n, m}^{ \pm}\right]_{1}\right) e_{2} .
\end{aligned}
$$

Also, from

$$
X_{n, m}^{ \pm}[\mu] e_{3}=\left[X_{n, m}^{ \pm}[\mu]\right]_{2} e_{1}-\left[X_{n, m}^{ \pm}[\mu]\right]_{1} e_{2}+\left[X_{n, m}^{ \pm}[\mu]\right]_{0} e_{3} .
$$

one sees that

$$
\begin{aligned}
Z_{n, m}^{ \pm}= & \left(a_{n, m} V_{n, m-1}^{\mp}+\frac{1}{n+m+2} V_{n, m+1}^{\mp}\right) e_{1} \\
& \pm\left(a_{n, m} V_{n, m-1}^{ \pm}-\frac{1}{n+m+2} V_{n, m+1}^{ \pm}\right) e_{2} .
\end{aligned}
$$

Theorem 4.2. The $n^{2}$ functions $\left\{Z_{k, m}^{ \pm}[\mu], Z_{k, 0}[\mu]\right\} \quad(1 \leq k \leq n, 1 \leq m \leq$ $k-1)$ form an orthogonal basis for $\mathcal{N}_{*}^{(n)}[\mu]$.

Proof. First we prove that $Z_{k, 0}[\mu]$ and $Z_{k, m}^{ \pm}[\mu]$ are indeed contragenic. As they have no scalar parts, it suffices to show that they are orthogonal to Vec $\mathcal{M}_{*}^{(n)}$. To do this, we use the basis obtained by dropping the scalar parts of the basis for $\mathcal{M}_{*}^{(n)}$ given in Theorem 3.2 . Since

$$
\left\{\Phi_{m_{1}}^{+}, \Phi_{m_{2}}^{-} \mid m_{1} \geq 0, m_{2} \geq 1\right\}
$$

is a system of orthogonal functions in $[0, \pi]$, then when $1 \leq m_{1} \leq k_{1}$ and $1 \leq m_{2} \leq k_{2}$, it is clear that

$$
\left\langle Z_{k_{1}, m_{1}}^{+}, \operatorname{Vec} X_{k_{2}, m_{2}}^{+}\right\rangle_{[\mu]}=\left\langle Z_{k_{1}, m_{1}}^{-}, \operatorname{Vec} X_{k_{2}, m_{2}}^{-}\right\rangle_{[\mu]}=0 .
$$




\begin{tabular}{|c|c|c|}
\hline$n$ & $m$ & $Z_{n, m}^{ \pm}$ \\
\hline 1 & 0 & $Z_{1,0}=-x_{2} e_{1}+x_{1} e_{2}$ \\
\hline & 0 & $Z_{2,0}=-3 x_{0} x_{2} e_{1}+3 x_{0} x_{1} e_{2}$ \\
\hline 2 & 1 & $\begin{aligned} Z_{2,1}^{+}= & 6 x_{1} x_{2} e_{1}+\frac{3}{30-20 \mu^{2}+6 \mu^{4}}\left(25 x_{2}^{2}-2 \mu^{2}-10 x_{2}^{2} \mu^{2}\right. \\
& +4 \mu^{4}+x_{2}^{2} \mu^{4}-2 \mu^{6}+10 x_{0}^{2}\left(-1+\mu^{2}\right)^{2} \\
& \left.+x_{1}^{2}\left(-35+30 \mu^{2}-11 \mu^{4}\right)\right) e_{2} \\
Z_{2,1}^{-}= & \frac{3}{30-20 \mu^{2}+6 \mu^{4}}\left(-35 x_{2}^{2}-2 \mu^{2}+30 x_{2}^{2} \mu^{2}+4 \mu^{4}\right. \\
& -11 x_{2}^{2} \mu^{4}-2 \mu^{6}+x_{1}^{2}\left(-5+\mu^{2}\right)^{2} \\
& \left.+10 x_{0}^{2}\left(-1+\mu^{2}\right)^{2}\right) e_{1}+6 x_{1} x_{2} e_{2}\end{aligned}$ \\
\hline \multirow{3}{*}{3} & 0 & $\begin{aligned} Z_{3,0}= & \frac{3}{14} x_{2}\left(-28 x_{0}^{2}+7 x_{1}^{2}+7 x_{2}^{2}+4 \mu^{2}\right) e_{1} \\
& -\frac{3}{14} x_{1}\left(-28 x_{0}^{2}+7 x_{1}^{2}+7 x_{2}^{2}+4 \mu^{2}\right) e_{2}\end{aligned}$ \\
\hline & 1 & $\begin{aligned} Z_{3,1}^{+}= & 30 x_{0} x_{1} x_{2} e_{1}+\frac{15 x_{0}}{70-84 \mu^{2}+30 \mu^{4}}\left(49 x_{2}^{2}\right. \\
& -6 \mu^{2}-42 x_{2}^{2} \mu^{2}+12 \mu^{4}+9 x_{2}^{2} \mu^{4}-6 \mu^{6}+14 x_{0}^{2}\left(-1+\mu^{2}\right)^{2} \\
& \left.+x_{1}^{2}\left(-91+126 \mu^{2}-51 \mu^{4}\right)\right) e_{2} \\
Z_{3,1}^{-}= & \frac{15 x_{0}}{70-84 \mu^{2}+30 \mu^{4}}\left(-91 x_{2}^{2}-6 \mu^{2}\right. \\
& +126 x_{2}^{2} \mu^{2}+12 \mu^{4}-51 x_{2}^{2} \mu^{4}-6 \mu^{6}+x_{1}^{2}\left(7-3 \mu^{2}\right)^{2} \\
& \left.+14 x_{0}^{2}\left(-1+\mu^{2}\right)^{2}\right) e_{1}+30 x_{0} x_{1} x_{2} e_{2}\end{aligned}$ \\
\hline & 2 & $\begin{aligned} Z_{3,2}^{+}= & -\frac{30 x_{2}}{35-14 \mu^{2}+3 \mu^{4}}\left(-21 x_{2}^{2}-2 \mu^{2}+14 x_{2}^{2} \mu^{2}+4 \mu^{4}\right. \\
& \left.-5 x_{2}^{2} \mu^{4}-2 \mu^{6}+x_{1}^{2}\left(-7+\mu^{2}\right)^{2}+14 x_{0}^{2}\left(-1+\mu^{2}\right)^{2}\right) e_{1} \\
& -\frac{30 x_{1}}{35-14 \mu^{2}+3 \mu^{4}}\left(49 x_{2}^{2}-2 \mu^{2}-14 x_{2}^{2} \mu^{2}+4 \mu^{4}+x_{2}^{2} \mu^{4}\right. \\
& \left.-2 \mu^{6}+14 x_{0}^{2}\left(-1+\mu^{2}\right)^{2}+x_{1}^{2}\left(-21+14 \mu^{2}-5 \mu^{4}\right)\right) e_{2} \\
Z_{3,2}^{-}= & \frac{60 x_{1}}{35-14 \mu^{2}+3 \mu^{4}}\left(28 x_{2}^{2}+\mu^{2}-14 x_{2}^{2} \mu^{2}-2 \mu^{4}+4 x_{2}^{2} \mu^{4}\right. \\
& \left.+\mu^{6}-7 x_{0}^{2}\left(-1+\mu^{2}\right)^{2}+x_{1}^{2}\left(-7+\mu^{4}\right)\right) e_{1} \\
& -\frac{60 x_{2}}{35-14 \mu^{2}+3 \mu^{4}}\left(-7 x_{2}^{2}+\mu^{2}-2 \mu^{4}+x_{2}^{2} \mu^{4}\right. \\
& \left.+\mu^{6}-7 x_{0}^{2}\left(-1+\mu^{2}\right)^{2}+2 x_{1}^{2}\left(14-7 \mu^{2}+2 \mu^{4}\right)\right) e_{2}\end{aligned}$ \\
\hline
\end{tabular}

Table 4: Spheroidal Contragenic Polynomials of Low Degree. 
On the other hand, when $m_{1}>0$ and $m_{2} \geq 0$, we have that

$$
\begin{aligned}
\left\langle Z_{k_{1}, m_{1}}^{ \pm}, \operatorname{Vec} X_{k_{2}, m_{2}}^{\mp}\right\rangle_{[\mu]}= & b_{k_{1}, m_{1}}^{-} \int_{\Omega_{\mu}}\left[X_{k_{1}, m_{1}}^{\mp}\right]_{1}\left[X_{k_{2}, m_{2}}^{\mp}\right]_{1} d x \\
& \mp b_{k_{1}, m_{1}}^{+} \int_{\Omega_{\mu}}\left[X_{k_{1}, m_{1}}^{ \pm}\right]_{2}\left[X_{k_{2}, m_{2}}^{\mp}\right]_{1} d x \\
& +b_{k_{1}, m_{1}}^{-} \int_{\Omega_{\mu}}\left[X_{k_{1}, m_{1}}^{\mp}\right]_{2}\left[X_{k_{2}, m_{2}}^{\mp}\right]_{2} d x \\
& \pm b_{k_{2}, m_{2}}^{+} \int_{\Omega_{\mu}}\left[X_{k_{1}, m_{1}}^{ \pm}\right]_{1}\left[X_{k_{2}, m_{2}}^{\mp}\right]_{2} d x
\end{aligned}
$$

where $b_{k, m}^{ \pm}$come from (29). Since the system

$$
\left\{\operatorname{Vec} X_{k, m}^{+}, \operatorname{Vec} X_{j, l}^{-} \mid 0 \leq k \leq n, 0 \leq m \leq k, 1 \leq j \leq n, 1 \leq l \leq j\right\}
$$

is orthogonal, straightforward computations show that

$$
\begin{aligned}
\left\langle Z_{k_{1}, m_{1}}^{ \pm}, \operatorname{Vec} X_{k_{2}, m_{2}}^{\mp}\right\rangle_{[\mu]}= & \frac{\pi}{2}\left(2 a_{k_{1}, m_{1}}\left(k_{1}+m_{1}+1\right) \int_{0}^{1 / \mu} \int_{0}^{\pi}\left(\widehat{V}_{k_{1}, m_{1}-1}\right)^{2} d u d v\right. \\
& -\frac{2}{\left(k_{1}+m_{1}+2\right)^{2}} \int_{0}^{1 / \mu} \int_{0}^{\pi}\left(\widehat{V}_{k_{1}, m_{1}+1}\right)^{2} d u d v \\
& \left.\mp \frac{2 \delta_{0, m_{1}}}{\left(k_{1}+2\right)^{2}} \int_{0}^{1 / \mu} \int_{0}^{\pi}\left(\widehat{V}_{k_{1}, 1}\right)^{2} d u d v\right) \delta_{m_{1}, m_{2}} \delta_{k_{1}, k_{2}} .
\end{aligned}
$$

Furthermore, using the expression (31) and recalling that

$$
\begin{aligned}
\operatorname{Vec} X_{k, m}^{-}= & \frac{1}{2}\left[\left((k+m+1) V_{k, m-1}^{-} \frac{1}{k+m+2} V_{k, m+1}^{-}\right) e_{1}\right. \\
& \left.+\left((k+m+1) V_{k, m-1}^{+}+\frac{1}{k+m+2} V_{k, m+1}^{+}\right) e_{2}\right]
\end{aligned}
$$


when $m>0$, we obtain that

$$
\begin{aligned}
\left\langle Z_{k, m}^{+}, \operatorname{Vec} X_{k, m}^{-}\right\rangle_{[\mu]}= & \frac{1}{2}\left[\int_{\Omega_{\mu}}\left(a_{k, m} V_{k, m-1}^{-}+\frac{1}{k+m+2} V_{k, m+1}^{-}\right)\right. \\
& \times\left((k+m+1) V_{k, m-1}^{-}-\frac{1}{k+m+2} V_{k, m+1}^{-}\right) d x \\
& +\int_{\Omega_{\mu}}\left(a_{k, m} V_{k, m-1}^{+}-\frac{1}{k+m+2} V_{k, m+1}^{+}\right) \\
& \left.\times\left((k+m+1) V_{k, m-1}^{+}+\frac{1}{k+m+2} V_{k, m+1}^{+}\right) d x\right] \\
= & a_{k, m}\left\|V_{k, m-1}^{+}\right\|_{[\mu]}^{2}-\frac{1}{(k+m+2)^{2}}\left\|V_{k, m+1}^{+}\right\|_{[\mu]}^{2} \\
= & 0
\end{aligned}
$$

by (28). Similarly, the orthogonality of $\left\{\Phi_{m}^{+}, \Phi_{l}^{-}\right\}$gives $\left\langle Z_{k, m}^{-}, \operatorname{Vec} X_{k, m}^{+}\right\rangle_{[\mu]}=$ 0 . Next, we expand

$$
\begin{aligned}
\left\langle Z_{k_{1}, 0}, \operatorname{Vec} X_{k_{2}, m}^{ \pm}\right\rangle_{[\mu]}= & \frac{1}{2\left(k_{1}+2\right)}\left(\left(k_{2}+m+1\right) \int_{\Omega_{\mu}} V_{k_{1}, 1}^{-} V_{k_{2}, m-1}^{ \pm} d x\right. \\
& -\frac{1}{k_{2}+m+2} \int_{\Omega_{\mu}} V_{k_{1}, 1}^{-} V_{k_{2}, m+1}^{ \pm} d x \\
& \pm\left(\left(k_{2}+m+1\right) \int_{\Omega_{\mu}} V_{k_{1}, 1}^{+} V_{k_{2}, m-1}^{\mp} d x\right. \\
& \left.\left.+\frac{1}{k_{2}+m+2} \int_{\Omega_{\mu}} V_{k_{1}, 1}^{+} V_{k_{2}, m+1}^{\mp} d x\right)\right) \\
= & 0
\end{aligned}
$$

again by orthogonality of $\left\{\Phi_{m}^{+}, \Phi_{l}^{-}\right\}$. For $k_{1} \neq k_{2}$, by the orthogonality of the system

$$
\begin{gathered}
\left\{V_{k_{1}, m_{1}}^{+}, V_{k_{2}, m_{2}}^{-} \mid 0 \leq k_{1} \leq n_{1}, 0 \leq k_{2} \leq n_{2},\right. \\
0 \leq m_{1} \leq k_{1}, 1 \leq m_{2} \leq k_{2} \\
\left.n_{1}, n_{2} \geq 0\right\}
\end{gathered}
$$


it remains to check that

$$
\begin{aligned}
\left\langle Z_{k, 0}, \operatorname{Vec} X_{k, 2}^{-}\right\rangle_{[\mu]} & =\frac{(k+m+1)}{2(k+2)}\left(\int_{\Omega_{\mu}}\left(V_{k, 1}^{-}\right)^{2} d x-\int_{\Omega_{\mu}}\left(V_{k, 1}^{+}\right)^{2} d x\right) \\
& =0
\end{aligned}
$$

the last equality is a consequence of

$$
\begin{aligned}
\int_{\Omega_{\mu}}\left(V_{k, 1}^{-}\right)^{2} d x & =\int_{0}^{\pi} \int_{0}^{1 / \mu}\left(\widehat{V}_{k, 1}\right)^{2} d v d u \int_{0}^{2 \pi} \sin ^{2} \phi d \phi \\
& =\int_{0}^{\pi} \int_{0}^{1 / \mu}\left(\widehat{V}_{k, 1}\right)^{2} d v d u \int_{0}^{2 \pi} \cos ^{2} \phi d \phi \\
& =\int_{\Omega_{\mu}}\left(V_{k, 1}^{+}\right)^{2} d x .
\end{aligned}
$$

We have verified that the functions $Z_{k, m}^{ \pm}$are contragenic.

It remains to prove the orthogonality of the system $\left\{Z_{k_{1}, m}^{ \pm}, Z_{k_{2}, 0}\right\}$. Using the expression (31), when $1 \leq m_{1}, m_{2}$ we have

$$
\begin{aligned}
\left\langle Z_{k_{1}, m_{1}}^{ \pm}, Z_{k_{2}, m_{2}}^{ \pm}\right\rangle_{[\mu]}= & a_{k_{1}, m_{1}} a_{k_{2}, m_{2}} \int_{\Omega_{\mu}} V_{k_{1}, m_{1}-1}^{\mp} V_{k_{2}, m_{2}-1}^{\mp} d x \\
& +\frac{a_{k_{1}, m_{1}}}{k_{2}+m_{2}+2} \int_{\Omega_{\mu}} V_{k_{1}, m_{1}-1}^{\mp} V_{k_{2}, m_{2}+1}^{\mp} d x \\
& +\frac{a_{k_{2}, m_{2}}}{k_{1}+m_{1}+2} \int_{\Omega_{\mu}} V_{k_{1}, m_{1}+1}^{\mp} V_{k_{2}, m_{2}-1}^{\mp} d x \\
& +\frac{1}{\left(k_{1}+m_{1}+2\right)\left(k_{2}+m_{2}+2\right)} \int_{\Omega_{\mu}} V_{k_{1}, m_{1}+1}^{\mp} V_{k_{2}, m_{2}+1}^{\mp} d x \\
& +a_{k_{1}, m_{1}} a_{k_{2}, m_{2}} \int_{\Omega_{\mu}} V_{k_{1}, m_{1}-1}^{ \pm} V_{k_{2}, m_{2}-1}^{ \pm} d x \\
& -\frac{a_{k_{1}, m_{1}}}{k_{2}+m_{2}+2} \int_{\Omega_{\mu}} V_{k_{1}, m_{1}-1}^{ \pm} V_{k_{2}, m_{2}+1}^{ \pm} d x \\
& -\frac{a_{k_{2}, m_{2}}}{k_{1}+m_{1}+2} \int_{\Omega_{\mu}} V_{k_{1}, m_{1}+1}^{ \pm} V_{k_{2}, m_{2}-1}^{ \pm} d x \\
& +\frac{1}{\left(k_{1}+m_{1}+2\right)\left(k_{2}+m_{2}+2\right)} \int_{\Omega_{\mu}} V_{k_{1}, m_{1}+1}^{ \pm} V_{k_{2}, m_{2}+1}^{ \pm} d x .
\end{aligned}
$$


Thus, by a similar argument to that used in the proof of Theorem 3.2,

$$
\begin{aligned}
\left\langle Z_{k_{1}, m_{1}}^{ \pm}, Z_{k_{2}, m_{2}}^{ \pm}\right\rangle_{[\mu]}= & 2 \pi \delta_{m_{1}, m_{2}} \delta_{k_{1}, k_{2}}\left(\left(a_{k_{1}, m_{1}}\right)^{2} \int_{0}^{\pi} \int_{0}^{1 / \mu}\left(\widehat{V}_{k_{1}, m_{1}-1}\right)^{2} d v d u\right. \\
& \left.+\left(\frac{1}{k_{1}+m_{1}+2}\right)^{2} \int_{0}^{\pi} \int_{0}^{1 / \mu}\left(\widehat{V}_{k_{1}, m_{1}+1}\right)^{2} d v d u\right) .
\end{aligned}
$$

On the other hand, when $1 \leq m \leq k_{1}$, we have that

$$
\begin{aligned}
\left\langle Z_{k_{1}, 0}, Z_{k_{2}, m}^{ \pm}\right\rangle_{[\mu]}= & \frac{1}{k_{1}+2}\left(a_{k_{2}, m} \int_{\Omega_{\mu}} V_{k_{1}, 1}^{-} V_{k_{2}, m-1}^{\mp} d x\right. \\
& +\frac{1}{k_{2}+m+2} \int_{\Omega_{\mu}} V_{k_{1}, 1}^{-} V_{k_{2}, m+1}^{\mp} d x \\
& \mp a_{k_{2}, m} \int_{\Omega_{\mu}} V_{k_{1}, 1}^{+} V_{k_{2}, m-1}^{ \pm} d x \\
& \left. \pm \frac{1}{\left(k_{2}+m+2\right)} \int_{\Omega_{\mu}} V_{k_{1}, 1}^{+} V_{k_{2}, m+1}^{ \pm} d x\right) .
\end{aligned}
$$

Then, it is clear that $\left\langle Z_{k_{1}, 0}, Z_{k_{2}, m}^{-}\right\rangle_{[\mu]}=0$. It remains to check that

$$
\left\langle Z_{k_{1}, 0}, Z_{k_{2}, m}^{+}\right\rangle_{[\mu]}=0,
$$

But this follows again from the formula for the cosine of a sum of angles and $\int_{0}^{2 \pi} \Phi_{m}^{+} d \phi=0$.

Finally, by the orthogonality of the system $\left\{\Phi_{m}^{ \pm}\right\}$,

$$
\left\langle Z_{k_{1}, m_{1}}^{ \pm}, Z_{k_{2}, m_{2}}^{\mp}\right\rangle_{[\mu]}=0 .
$$

\subsection{Further observations}

Subspaces analogous to the homogeneous polynomials are obtained by defining $\widehat{H a r}^{(n)}(\Omega)$ to be the orthogonal component of $\operatorname{Har}_{*}^{(n-1)}(\Omega)$ in $\operatorname{Har}_{*}^{(n)}(\Omega)$, so we have an orthogonal decomposition

$$
\operatorname{Har}_{2}(\Omega)=\bigoplus_{n=0}^{\infty} \widehat{\operatorname{Har}}^{(n)}(\Omega)
$$


(For $\Omega=\Omega_{0}$ this is in fact the decomposition by spherical harmonics.) Similarly, let $\widehat{\mathcal{N}}^{(n)}[\mu]$ be the orthogonal component of $\mathcal{N}_{*}^{(n-1)}[\mu]$ in $\mathcal{N}_{*}^{(n)}[\mu]$, so $\mathcal{N}_{*}^{(n)}[\mu]=\bigoplus_{k=1}^{n} \widehat{\mathcal{N}}^{(k)}[\mu]$. Thus

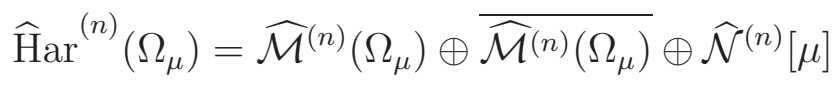

where the monogenic part $\widehat{\mathcal{M}}^{(n)}\left(\Omega_{\mu}\right)$ is defined analogously.

Natural linear mappings from the space of spherical harmonics $\operatorname{Har}^{(n)}\left(\Omega_{0}\right)$ to the spheroidal $\operatorname{Har}^{(n)}\left(\Omega_{\mu}\right)$ were worked out in [4]. These correspondences will be studied in the context of monogenic functions in future work.

Theorem 4.3. The functions $Z_{k, m}^{ \pm}[\mu]$ span a dense set in $\mathcal{N}\left(\Omega_{\mu}\right)$. Therefore the functions $Y_{k, m}^{ \pm \pm}, Z_{k, m}^{ \pm}[\mu]$ form an orthogonal basis for $\operatorname{Har}_{2}\left(\Omega_{\mu}\right)$.

Proof. Let $Z \in \mathcal{N}\left(\Omega_{\mu}\right)$. Write $Z=\sum_{k=0}^{\infty} U_{k}$, where $U_{k} \in \widehat{\operatorname{Har}}^{(k)}\left(\Omega_{\mu}\right)$, and let $U_{k}=Y_{k}+Z_{k}$ be the decomposition into ambigenic and contragenic polynomials. Thus $Z=Y+\sum_{1}^{\infty} Z_{k}$ where $Y=\sum_{0}^{\infty} Y$ is both ambigenic and contragenic, i.e. $Y=0$. Hence $Z \in \bigoplus \widehat{\mathcal{N}}^{(k)}[\mu]$ as required.

The orthogonal decomposition $\left(\mathcal{M}_{2}(\Omega)+\overline{\mathcal{M}}_{2}(\Omega)\right) \oplus \mathcal{N}(\Omega)$ justifies the idea of referring to the "ambigenic part" or the "monogenic part" of any harmonic function $\Omega \rightarrow \mathbb{R}^{3}$ (the latter being determined up to an additive monogenic constant). Theorem 4.3 provides a method of calculation of this part in the case of spheroids $\Omega_{\mu}$, by obtaining the Fourier coefficients as in any Hilbert space, and then discarding the contragenic and antimonogenic terms.

As a consequence of the fact that the norm on $L_{2}\left(\Omega_{\mu}\right)$ depends upon $\mu$, the spaces $\mathcal{N}\left(\Omega_{\mu}\right)$ are distinct for distinct values of $\mu$. It is easy to see that

Proposition 4.4. $\mu \neq \mu^{\prime}$ implies $\mathcal{N}\left(\Omega_{\mu}\right) \neq \mathcal{N}\left(\Omega_{\mu^{\prime}}\right)$.

Indeed, consider the polynomials of degree 2, which are of the form

$$
a_{0} Z_{2,0}[\mu]+a_{+} Z_{2,1}^{+}[\mu]+a_{-} Z_{2,1}^{-}[\mu]
$$

for real $a_{0}, a_{+}, a_{-}$. From Table 4 we see that the coefficients of $x_{1} x_{2}$ and $x_{2}^{2}$ are, respectively, $6 a_{+}$and $\left(75 a_{+}-105 a_{-}\right) /\left(30-20 \mu^{2}+6 \mu^{4}\right)$. These coefficients determine $a_{+}$and $a_{-}$, and then $a_{0}$ is determined by the coefficient of $x_{0} x_{1}$. Such a polynomial determines the value of $\mu \in \mathbb{R}^{+} \cup i \mathbb{R}^{+}$, and thus can be in only one space $\mathcal{N}\left(\Omega_{\mu}\right)$. 
The fact that the notion of contragenicity depends on the domain implies that it is not a local property, in contrast to harmonicity and monogenicity. In particular, any attempt to seek a condition on the derivatives of a harmonic function to detect whether it is monogenic or not is doomed to failure. It is not known, however, whether such a condition may exist associated to a fixed domain, such as a sphere or spheroid.

\section{Acknowledgements}

The first author's work is supported by CONACyT Grant 1600594. The second author acknowledges financial support by the Asociación Mexicana de Cultura, A. C.

\section{References}

[1] C. Álvarez-Peña, R. M. Porter, (2014), Contragenic functions of three variables, Complex Anal. Oper. Theory, Vol. 8, No. 2, 409-427.

[2] F. Brackx, R. Delanghe and F. Sommen, (1982), Clifford analysis, Pitman Advanced Publishing Program.

[3] S.Bock And K. GÜrLebeck, (2010), On a generalized Appell system and monogenic power series, Mathematical Methods in the Applied Sciences 33 394-411.

[4] H.A. Buchdahl, N.P. Buchdahl and P.J. Stiles, (1977), On a relation between spherical and spheroidal harmonics, J. Phys. A: Math. Gen. 10:11.

[5] I. CaÇÃo, K. Gürlebeck and H. MaloneK, (2001), Special monogenic polynomials and $L_{2}$-approximation, Advances in Applied Clifford Algebras 11 47-60.

[6] I. CAÇÃO, K. GÜrleBeCK And S. BoCK, (2004), Complete orthonormal systems of spherical monogenics - a constructive approach, Methods of Complex and Clifford Analysis (Proceedings of ICAM Hanoi 2004), L.H. Son, W. Tutschke and S. Jain, eds., SAS International Publications.

[7] I. CAÇÃ̃o, (2004), Constructive approximation by monogenic polynomials, doctoral dissertation, Universidade de Aveiro. 
[8] I. CAÇÃO, (2010), Complete orthonormal sets of polynomial solutions of the Riesz and Moisil-Teodorescu systems in $\mathbb{R}^{3}$, Numerical Algorithms, Vol. 55, No. 2-3, 191-203.

[9] R. Delanghe, (2007), On homogeneous polynomial solutions of the Riesz system and their harmonic potentials, Complex Variables and Elliptic Equations, Vol. 52, No. 10-11, 1047-1062.

[10] R. Delanghe, (2009), On homogeneous polynomial solutions of the Moisil-Théodoresco system in $\mathbb{R}^{3}$, Computational Methods and Function Theory, Vol. 9, No. 1, 199-212.

[11] P. Garabedian, (1953), Orthogonal harmonic polynomials, Pacific J. Math. Vol. 3, No. 3, 585-603.

[12] K. Gürlebeck, K. Habetha And W. Sprössig, (2008), Holomorphic functions in the plane and n-dimensional space, Birkhäuser Verlag, Basel-Boston-Berlin. Contemp. Math. 212, 95-107.

[13] E. Hobson, (1931), The theory of spherical and ellipsoidal harmonics, Cambridge.

[14] H. Leutwiler, (2001), Quaternionic analysis in $\mathbb{R}^{3}$ versus its hyperbolic modification, en: Brackx, F., Chisholm, J.S.R., Soucek, V. (eds.) Proceedings of the NATO Advanced Research Workshop held in Prague, October 30 - November 3, 2000 25, Kluwer Academic Publishers, Dordrecht, Boston, London.

[15] J. MoraIs, (2009), Approximation by homogeneous polynomial solutions of the Riesz system in $\mathbb{R}^{3}$, Ph.D. thesis (Bauhaus-Universitãt Weimar).

[16] J. Morais, (2011), A complete orthogonal system of spheroidal monogenics, Journal of Numerical Analysis, Industrial and Applied Mathematics (JNAIAM) 6 105-119.

[17] J. Morais And K. GürleBeCK, (2012), Real-part estimates for solutions of the Riesz system in $\mathbb{R}^{3}$, Complex Var. Elliptic Equ. 57:5, 505522 . 
[18] J. Morais And K. GürLEBECK, (2012), Bloch's theorem in the context of quaternion analysis, Computational Methods and Function Theory, Vol. 12, No. 2, 541-558.

[19] J. Morais, K. Avetisyan and K. Gürlebeck, (2013), On Riesz systems of harmonic conjugates in $\mathbb{R}^{3}$, Mathematical Methods in the Applied Sciences, Vol. 36, No. 12, 1598-1614.

[20] J. Morais, (2013), An orthogonal system of monogenic polynomials over prolate spheroids in $\mathbb{R}^{3}$, Mathematical and Computer Modelling $57,425-434$.

[21] J. Morais, K.I. Kou And W. Sprössig, (2013), Generalized holomorphic Szegö kernel in 3D spheroids, Computers and Mathematics with Applications 65, 576-588.

[22] J. Morais, (2014), Computational aspects of the continuum quaternionic wave functions for hydrogen, Annals of Physics, Vol. 349, 171188.

[23] J. Morais And I. CAÇÃo, (2015), Quaternion Zernike spherical polynomials, Mathematics of Computation, Vol. 84, No. 293, 1317-1337.

[24] J. Morais, M.A. PÉrez-De la Rosa And K.I. Kou, (2015), Computational geometric and boundary value properties of oblate spheroidal quaternionic wave functions, Wave Motion, Vol. 57, 112-128.

[25] J. Morais And M.A. PÉRez-De LA RosA, (2015), Towards a quaternionic function theory linked with the Lamé's wave functions, Mathematical Methods in the Applied Sciences, Vol. 38, No. 17, 4365-4387.

[26] J. Morais, M.H. Nguyen And K.I. Kou, (2016), On 3D orthogonal prolate spheroidal monogenics, Mathematical Methods in the Applied Sciences Vol. 39, No. 4, 635-648.

[27] J. Morais AND K.I. Kou, (2016), Constructing prolate spheroidal quaternion wave functions on the sphere, Mathematical Methods in the Applied Sciences, Vol. 39, No. 14, 3961-3978.

[28] C. Müller, (1966), Spherical Harmonics, Lectures Notes in Mathematics 17 Berlin: Springer - Verlag. 
[29] H.M. Nguyen, K. Gürlebeck, J. Morais and S. Bock, (2014), On orthogonal monogenics in oblate spheroidal domains and recurrence formulae, Integral Transforms Spec. Funct., Vol. 25, No. 7, 513-527.

[30] A. Sudbery, (1979), Quaternionic analysis, Math. Proc. Cambridge Phil. Soc. 85, 199-225.

[31] C. Zou, K.I. Kou And J. Morais, Prolate spheroidal wave functions associated with the quaternionic Fourier transform, Mathematical Methods in the Applied Sciences, DOI: 10.1002/mma.4439. 